\title{
Assembly and Transfer of Iron-Sulfur Clusters in the Plastid
}

\author{
Yan $L u^{*}$ \\ Department of Biological Sciences, Western Michigan University, Kalamazoo, MI, United States
}

Iron-Sulfur (Fe-S) clusters and proteins are essential to many growth and developmental processes. In plants, they exist in the plastids, mitochondria, cytosol, and nucleus. Six types of Fe-S clusters are found in the plastid: classic 2Fe-2S, NEET-type 2Fe-2S, Rieske-type 2Fe-2S, 3Fe-4S, 4Fe-4S, and siroheme 4Fe-4S. Classic, NEET-type, and Rieske-type 2Fe-2S clusters have the same 2Fe-2S core; similarly, common and siroheme $4 \mathrm{Fe}-4 \mathrm{~S}$ clusters have the same $4 \mathrm{Fe}-4 \mathrm{~S}$ core. Plastidial Fe-S clusters are assembled by the sulfur mobilization (SUF) pathway, which contains cysteine desulfurase (EC 2.8.1.7), sulfur transferase (EC 2.8.1.3), Fe-S scaffold complex, and Fe-S carrier proteins. The plastidial cysteine desulfurase-sulfur transferase-Fe-S-scaffold complex system is responsible for de novo assembly of all plastidial Fe-S clusters. However, different types of Fe-S clusters are transferred to recipient proteins via respective Fe-S carrier proteins. This review focuses on recent discoveries on the molecular functions of different assembly and transfer factors involved in the plastidial SUF pathway. It also discusses potential points for regulation of the SUF pathway, relationships among the

OPEN ACCESS

Edited by: Fei Yu,

Northwest A\&F University, China

Reviewed by:

Aigen Fu,

Northwest University, China Stefano Santabarbara, Consiglio Nazionale Delle Ricerche (CNR), Italy

*Correspondence: Yan Lu

yan.1.1u@wmich.edu

Specialty section: This article was submitted to

Plant Physiology, a section of the journal Frontiers in Plant Science

Received: 03 October 2017 Accepted: 28 February 2018 Published: 14 March 2018

Citation:

Lu Y (2018) Assembly and Transfer of Iron-Sulfur Clusters in the Plastid.

Front. Plant Sci. 9:336.

doi: $10.3389 /$ fpls.2018.00336 plastidial, mitochondrial, and cytosolic Fe-S assembly and transfer pathways, as well as several open questions about the carrier proteins for Rieske-type 2Fe-2S, NEET-type 2Fe-2S, and 3F-4S clusters.

Keywords: iron-sulfur cluster, cysteine desulfurase, sulfur transferase, iron-sulfur scaffold complex, iron-sulfur carrier protein

\section{INTRODUCTION}

Iron-Sulfur (Fe-S) clusters are sulfide $\left(\mathrm{S}^{2-}\right)$-linked di-iron, tri-iron, or tetra-iron clusters found in metalloproteins. Iron $(\mathrm{Fe})$ is a transition metal, which can form cations with an incomplete $d$ subshell. This property makes $\mathrm{Fe}$ show a variable valency (e.g., $\mathrm{Fe}^{2+}$ and $\mathrm{Fe}^{3+}$ ) and the ability to form coordination units, such as Fe-S clusters. Depending on the ligands, organic structures, and protein folds, the redox potential of Fe-containing cofactors may range between -650 and $+450 \mathrm{mV}$ (Beinert, 2000).

Due to the varying redox potential of Fe, Fe-S clusters have the ability to transfer electrons, especially when they are arranged sequentially with individual distances of $<14 \AA$ (Balk and Schaedler, 2014). Fe-S clusters are best known for participating in oxidation-reduction reactions in photosynthetic electron transport in thylakoid membranes and respiratory electron transport in the inner mitochondrial membrane (Johnson et al., 2005; Balk and Pilon, 2011; Couturier et al., 2013). Examples of Fe-S proteins involved in photosynthetic electron transport include the photosynthetic electron transfer $\mathrm{C}$ (PetC) protein in the cytochrome $b_{6} f$ complex, Photosystem I (PSI) core subunits PsaA, PsaB, and PsaC, and ferredoxins (Balk and Pilon, 2011). Examples of Fe-S complexes involved in respiratory electron transport include NADH dehydrogenase (Complex I, EC 1.6.99.3), succinate dehydrogenase (Complex II, EC 1.3.5.1), and cytochrome $b c 1$ complex (Complex III, EC 1.10.2.2) (Couturier et al., 2013). 


\section{COMMON TYPES OF Fe-S CLUSTERS AND EXAMPLES OF Fe-S PROTEINS IN THE PLASTID}

Common types of Fe-S clusters found in the plastid include (1) classic $2 \mathrm{Fe}-2 \mathrm{~S}$ coordinated by four cysteine (Cys) residues; (2) NEET-type $2 \mathrm{Fe}-2 \mathrm{~S}$ coordinated by three Cys and one His residues; (3) Rieske-type $2 \mathrm{Fe}-2 \mathrm{~S}$ coordinated by two Cys and two His residues; (4) $3 \mathrm{Fe}-4 \mathrm{~S}$ coordinate by three Cys residues; (5) $4 \mathrm{Fe}-$ $4 \mathrm{~S}$ coordinated by four Cys residues (or three Cys residues and the hydroxide group from water); and (6) $4 \mathrm{Fe}-4 \mathrm{~S}$ coordinated by four Cys residues with one Cys cross-bridging a siroheme (Figure 1) (Johnson et al., 2005; Balk and Pilon, 2011; Couturier et al., 2013; Balk and Schaedler, 2014). 3Fe-4S and 4Fe-4S clusters are cubane-type clusters. The three $2 \mathrm{Fe}-2 \mathrm{~S}$ clusters are rhombictype clusters, containing the same $2 \mathrm{Fe}-2 \mathrm{~S}$ core. Therefore, the relative abundances of the three rhombic-type clusters depend not on the rate of cluster assembly, but on the availability of appropriate carriers and recipient proteins.

The plant-type ferredoxin $(\mathrm{Fd})$ is a classic $2 \mathrm{Fe}-2 \mathrm{~S}$ protein. It is a small and soluble protein with four conserved Cys residues capable of ligating one 2Fe-2S cluster (Hanke and Mulo, 2013). In the chloroplast stroma, $\mathrm{Fd}$ acts as a mobile electron carrier during photosynthetic electron transport, carrying electrons from PSI to PSI-associated $\mathrm{Fd}^{-\mathrm{NADP}^{+}}$reductase, for the reduction of $\mathrm{NADP}^{+}$to NADPH (Hase et al., 2006). The plant-type Fd also serves as an electron donor to a number of chloroplast stromal proteins, such as nitrite reductase (NiR, EC 1.7.7.1), sulfite reductase (SiR, EC 1.8.7.1), and Fd-dependent glutamine:2oxyoglutarate aminotransferases (Fd-GOGATs, EC 1.4.7.1) (Hase et al., 2006). Whether Fd serves as an electron carrier or electron donor, the electron is actually carried by the $2 \mathrm{Fe}-2 \mathrm{~S}$ cluster within the protein (Hase et al., 2006).

Abbreviations: ABA, abscisic acid deficient 3; ABC, ATP binding cassette; ACO, aconitase; APR1, adenosine $5^{\prime}$-phosphosulfate reductase 1; At, Arabidopsis thaliana; ATPase, adenosine triphosphatase; BIO, biotin synthase; BolA, DNAbinding transcriptional regulator BolA; CAO, chlorophyllide a oxygenase; CIA, cytosolic iron-sulfur pathway; COG0354m and COG0354p, mitochondrial and plastidial Clusters of Orthologous Groups 0354 proteins; CpNifS, chloroplastic nitrogen fixation S-like; CpIscA1, chloroplast iron-sulfur cluster protein A1; CpSufS, chloroplastic sulfur mobilization protein S; DUF59 and DUF971, domain of unknown function 59 and 971; Fd, ferredoxin; Fd-GOGAT1 and Fd-GOGAT2, ferredoxin-dependent Gln oxoglutarate aminotransferase 1 and 2; FER1, FER2, and FER3, ferritin 1, 2, and 3; FNR, fumarate and nitrate reduction regulatory protein; FTR, ferredoxin-thioredoxin reductase; GFP, green fluorescent protein; GIY-YIG, GlyIleTyr-TyrIleGly; GRX, glutaredoxin; GRXS14 and GRXS16, glutaredoxin S14 and S16; HCAR, 7-hydroxymethyl chlorophyll a reductase; HCF101, high chlorophyll fluorescence 101; IRP1, iron regulatory protein 1; ISC, iron-sulfur cluster pathway; IscS, iron-sulfur cluster protein S; ISU1, iron sulfur cluster assembly protein 1; LEU1, 3-isopropylmalate isomerase 1; LIP, lipoic acid synthase; MiaB, isopentenyl-adenosine A37 tRNA methylthiolase; NadA, quinolinate synthase; NAP1, NAP6, and NAP7, non-intrinsic ABC protein 1, 6, and 7; NFU1, NFU2, and NFU3, nitrogen fixation subunit $\mathrm{U} 1,2$, and 3; NFS1/NifS1 and NFS2, nitrogen fixation S-like 1 and 2; NiR, nitrite reductase; NTPase, nucleotide phosphatase; PetC, photosynthetic electron transfer C; PAO, pheophorbide $a$ oxygenase; PSI, Photosystem I; PsaA, PsaB, and PsaC, Photosystem I proteins A, B, and C; RNAi, RNA interference; SiR, sulfite reductase; SUF, sulfur mobilization pathway; SufA1, SufB, SufC, SufD, SufE1, SufE2, and SufE3, sulfur mobilization protein A1, B, C, D, E1, E2, and E3; TP, transit peptide; YFP, yellow fluorescent protein.
One example of NEET-type $2 \mathrm{Fe}-2 \mathrm{~S}$ proteins is At-NEET, a CDGSH motif-containing Arabidopsis thaliana protein dually targeted to the chloroplast stroma and mitochondria (Nechushtai et al., 2012; Su et al., 2013). The CDGSH motif contains a 16-amino acid consensus sequence $\left(\underline{\mathrm{C}}-\mathrm{X}-\underline{\mathrm{C}}-\mathrm{X}_{2}-[\mathrm{S} / \mathrm{T}]-\mathrm{X}_{3}-\mathrm{P}-\mathrm{X}\right.$ C-D-G-[S/A/T]- $\underline{H}$, where the three $\mathrm{Cys}\left[\mathrm{C}^{74}, \mathrm{C}^{76}\right.$, and $\left.\mathrm{C}^{85}\right]$ residues and one His $\left[\mathrm{H}^{89}\right]$ residue for NEET-type $2 \mathrm{Fe}-2 \mathrm{~S}$ cluster coordination are underlined). The recombinant At-NEET homodimer coordinates two labile $2 \mathrm{Fe}-2 \mathrm{~S}$ clusters, which are readily transferred to apo Fd in in vivo assays (Nechushtai et al., 2012). Therefore, Nechushtai et al. (2012) proposed that ATNEET may serve as a NEET-type $2 \mathrm{Fe}-2 \mathrm{~S}$ carrier for plastidial and mitochondrial $\mathrm{Fe}-\mathrm{S}$ assembly and transfer pathways. Compared to classic $2 \mathrm{Fe}-2 \mathrm{~S}$, NEET-type $2 \mathrm{Fe}-2 \mathrm{~S}$ is relatively unstable due to its atypical coordination with three Cys and one His residues (Wiley et al., 2007). Protonation of the ligating His residue could trigger cluster release, indicating that His ligation is also important for the $\mathrm{pH}$ lability of NEET-type $2 \mathrm{Fe}-2 \mathrm{~S}$ (Wiley et al., 2007).

PetC is an example of Rieske-type $2 \mathrm{Fe}-2 \mathrm{~S}$ proteins. The Rieske-type $2 \mathrm{Fe}-2 \mathrm{~S}$ cluster in PetC is essential to photosynthetic electron transport. It accepts electrons from plastoquinol and transfers to the heme Fe of the cytochrome $f$ protein (Madueño et al., 1992). Riesk-type $2 \mathrm{Fe}-2 \mathrm{~S}$ proteins contain a Riesketype $2 \mathrm{Fe}-2 \mathrm{~S}$-binding domain $\left(\underline{\mathrm{CX}} \underline{\mathrm{HXGCX}}{ }_{12-44} \mathrm{CXC} \underline{\mathrm{H}}\right.$, where the two Cys and two His residues for cluster coordination are underlined) (Link, 1999). The asymmetric coordination pattern (Figure 1C) of Rieske-type $2 \mathrm{Fe}-2 \mathrm{~S}$ results in distinctive redox and spectroscopic properties (Kounosu et al., 2004). Compared to classic $2 \mathrm{Fe}-2 \mathrm{~S}$, Rieske-type $2 \mathrm{Fe}-2 \mathrm{~S}$ has a relatively positive midpoint redox potential and its visible spectrum is red-shifted (Mason and Cammack, 1992).

Fd-GOGATs are examples of $3 \mathrm{Fe}-4 \mathrm{~S}$ proteins. Plants have two Fd-GOGAT isoforms: Fd-GOGAT1 and Fd-GOGAT2 (Coschigano et al., 1998). Fd-GOGAT1 is expressed in leaf chloroplasts and its primary role is photorespiration and nitrogen assimilation in leaves; Fd-GOGAT2 is expressed in root plastids and its primary role is nitrogen assimilation in roots (Coschigano et al., 1998). Fd-GOGATs function via non-covalent binding of $\mathrm{Fd}$ and subsequent delivery of reducing equivalents from $\mathrm{Fd}$ to FMN (another cofactor) via the 3Fe-4S cluster (van den Heuvel et al., 2002). Both FMN and $3 \mathrm{Fe}-4 \mathrm{~S}$ are located in the catalytic centers of Fd-GOGATs (van den Heuvel et al., 2002).

PSI core proteins $\mathrm{PsaA}, \mathrm{PsaB}$, and $\mathrm{PsaC}$ are examples of $4 \mathrm{Fe}$ $4 \mathrm{~S}$ proteins. PSI has three $4 \mathrm{Fe}-4 \mathrm{~S}$ clusters, each coordinated by four Cys residues. One is known as $\mathrm{F}_{\mathrm{X}}$, which is bound to the $\mathrm{PsaA} / \mathrm{PsaB}$ heterodimer. The other two are known as $\mathrm{F}_{\mathrm{A}}$ and $\mathrm{F}_{\mathrm{B}}$, both are bound to PsaC (Saenger et al., 2002). These $4 \mathrm{Fe}-4 \mathrm{~S}$ clusters are essential to photosynthetic electron transport: they serve as sequential electron carriers $\left(\mathrm{F}_{\mathrm{X}} \rightarrow \mathrm{F}_{\mathrm{A}} \rightarrow \mathrm{F}_{\mathrm{B}}\right)$ within PSI.

$\mathrm{NiR}$ and $\mathrm{SiR}$, two enzymes catalyzing the six electron reduction of nitrite and sulfite respectively (Raux-Deery et al., 2005), are siroheme $4 \mathrm{Fe}-4 \mathrm{~S}$ proteins. The active site of these enzymes has a siroheme attached to the $4 \mathrm{Fe}-4 \mathrm{~S}$ cluster via a Cys residue (Crane et al., 1995; Crane and Getzoff, 1996). Therefore, the siroheme $4 \mathrm{Fe}-4 \mathrm{~S}$ cluster is central to the reductive activity of $\mathrm{NiR}$ and $\mathrm{SiR}$. The insertion of $\mathrm{Fe}$ into siroheme is carried out by 


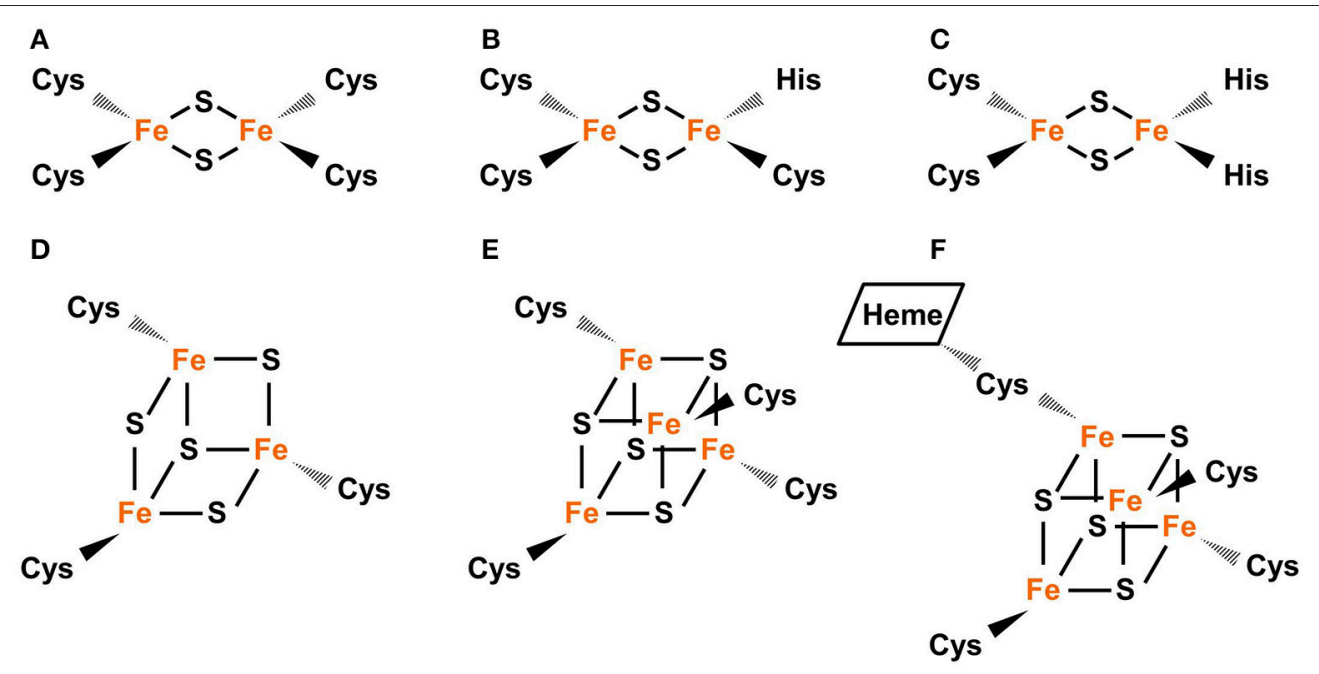

FIGURE 1 | Common types of Fe-S clusters found in the plastid. (A) Classic 2Fe-2S coordinated by four Cys residues, as in the plant-type Fd (ferredoxin). (B) NEET-type 2Fe-2S coordinated by three Cys and one His residues, as in NEET. (C) Rieske-type 2Fe-2S coordinated by two Cys and two His residues, as in PetC (photosynthetic electron transfer C). (D) 3Fe-4S coordinated by three Cys residues as in Fd-GOGATs (ferredoxin-dependent Gln oxoglutarate aminotransferases). (E) 4Fe-4S coordinated by four Cys residues, as in PsaA, PsaB, and PsaC (Photosystem I proteins A-C). (F) 4Fe-4S coordinated by four Cys residues with a thiolate ligand serving also for siroheme, as in NiR (nitrite reductase) and SiR (sulfite reductase). In Fe-S clusters, the oxidation state of Fe could be +2 or +3 while the oxidation state of $\mathrm{S}$ is -2 .

sirohydrochlorin ferrochelatase (EC 4.99.1.4), which contains a 2Fe-2S cluster (Saha et al., 2012).

In Fe-S clusters, $\mathrm{Fe}^{2+}$ or $\mathrm{Fe}^{3+}$ is combined with sulfide $\left(\mathrm{S}^{2-}\right)$. The valency of the entire cluster could be indicated with square brackets (e.g., $[2 \mathrm{Fe}-2 \mathrm{~S}]^{2+}$, in which both $\mathrm{Fe}$ ions exist as $\mathrm{Fe}^{3+}$ ). After $\mathrm{S}^{2-}$ is incorporated into the cluster, it does not undergo redox transitions. However, $\mathrm{Fe}^{3+}$ in the cluster may be reduced to $\mathrm{Fe}^{2+}$ and vice versa. For example, $[2 \mathrm{Fe}-2 \mathrm{~S}]^{2+}$ may receive an electron and become reduced to $[2 \mathrm{Fe}-2 \mathrm{~S}]^{1+}$ and $[2 \mathrm{Fe}-2 \mathrm{~S}]^{1+}$ may lose an electron and become oxidized to $[2 \mathrm{Fe}-2 \mathrm{~S}]^{2+}$.

Different types of Fe-S clusters are inter-convertible. [4Fe$4 \mathrm{~S}]^{2+}$ (a common oxidation state of $4 \mathrm{Fe}-4 \mathrm{~S}$ ) could be converted to $[2 \mathrm{Fe}-2 \mathrm{~S}]^{2+}$ (a common oxidation state of $2 \mathrm{Fe}-2 \mathrm{~S}$ ) via oxidative cleavage (Holm and Lo, 2016). For example, [4Fe$4 \mathrm{~S}]^{2+}$-containing fumarate and nitrate reduction regulatory protein (FNR) is a DNA-binding homodimer under anaerobic conditions (Zhang et al., 2012). Under high oxygen, the [4Fe$4 \mathrm{~S}]^{2+}$ cluster is quickly converted to a classic $[2 \mathrm{Fe}-2 \mathrm{~S}]^{2+}$ cluster, with the release of one $\mathrm{Fe}^{3+}$ ion, one $\mathrm{Fe}^{2+}$ ion, two $\mathrm{S}^{2-}$ ions, and a superoxide ion $\left(\mathrm{O}_{2}^{-}\right)$, via a two-step process (Crack et al., 2008; Zhang et al., 2012). In the first step, [4Fe-4S $]^{2+}$ is oxidized by one electron from molecular oxygen, producing an intermediate $[3 \mathrm{Fe}-4 \mathrm{~S}]^{1+}$, an $\mathrm{Fe}^{2+}$ ion, and $\mathrm{a} \mathrm{O}_{2}^{-}$ion. In the second step, the intermediate $[3 \mathrm{Fe}-4 \mathrm{~S}]^{1+}$ converts spontaneously to $[2 \mathrm{Fe}-2 \mathrm{~S}]^{2+}$, releasing an $\mathrm{Fe}^{3+}$ ion and two $\mathrm{S}^{2-}$ ions. This two-step conversion causes the transition of FNR from dimer to monomer and the loss of DNA-binding ability. The [4Fe$4 \mathrm{~S}]^{2+}$-to- $[2 \mathrm{Fe}-2 \mathrm{~S}]^{2+}$ conversion could be reversed via reductive coupling. For example, two $[2 \mathrm{Fe}-2 \mathrm{~S}]^{2+}$ clusters in FNR could receive two electrons and be reverted to $[4 \mathrm{Fe}-4 \mathrm{~S}]^{2+}$ after incubation with DTT under anaerobic conditions (Zhang et al., 2012).
$\mathrm{Fe}-\mathrm{S}$ clusters are sensitive to oxygen and reactive oxygen species (ROS) (Couturier et al., 2013; Balk and Schaedler, 2014) and the assembly of Fe-S clusters is influenced by the availability of Fe and S (Vigani et al., 2009). The inter-convertibility among different types of Fe-S clusters may represent a route to repair damaged $\mathrm{Fe}-\mathrm{S}$ clusters and/or a regulatory process in response to changes in cellular and external environments, especially redox status. As discussed below, loss-of-function mutations in a specific plastidial $\mathrm{Fe}-\mathrm{S}$ carrier protein do not result in uniform reductions in the levels of different types of plastidial $\mathrm{Fe}-\mathrm{S}$ clusters. These observations suggest that cluster inter-conversion may not be sufficient to compensate for the loss of a specific Fe-S carrier protein.

\section{Fe-S ASSEMBLY AND TRANSFER PATHWAYS IN PLANTS}

The Fe-S assembly and transfer process can be separated into two sequential steps (Figure 2). The first step is the assembly of Fe-S clusters on a scaffold complex by Cys desulfurase (EC 2.8.1.7), with the help from sulfur transferase (EC 2.8.1.3). The second step is the transfer of $\mathrm{Fe}-\mathrm{S}$ clusters from the scaffold complex to recipient proteins via carrier proteins. The initial identification of proteins involved in $\mathrm{Fe}-\mathrm{S}$ assembly was from the analysis of proteins required for nitrogen fixation in Gram-negative bacterium Azotobacteria vinelandii (Frazzon and Dean, 2003; Dos Santos et al., 2004). The work on the synthesis of nitrogenase (molybdenum [Mo])-Fe-S cofactors led to the discovery of the NIF (nitrogen fixation) system and established the requirement of Cys desulfurase, sulfur transferase, and Fe-S scaffold protein(s) during Fe-S assembly (Frazzon and Dean, 2003). While the NIF 


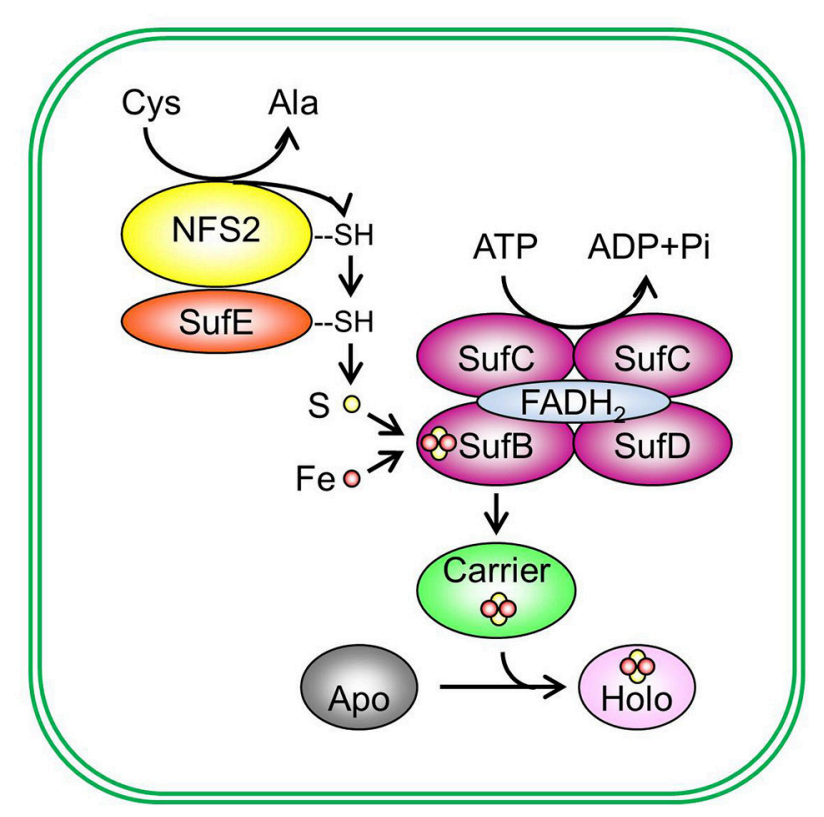

FIGURE 2 | De novo assembly and transfer of Fe-S clusters in the plastid. In the plastid, Cys desulfurase NFS2 (nitrogen fixation S-like 2) removes sulphane $\left(\mathrm{S}^{0}\right)$ from Cys and converts cysteine (Cys) to alanine (Ala). Sulphane $\left(\mathrm{S}^{0}\right)$ is transferred from Cys desulfurase to sulfur transferase SufE (sulfur mobilization protein $\mathrm{E}$ ) and then to SufB (sulfur mobilization protein B) of the $\mathrm{SufBC}_{2} \mathrm{D}$ scaffold complex. On the $\mathrm{SufBC}_{2} \mathrm{D}$ scaffold complex, whose function requires cofactor $F A D H_{2}$, sulphane $\left(S^{0}\right)$ is reduced to sulfide $\left(S^{2-}\right)$ and is incorporated in Fe-S clusters. The SufBC 2 D complex has ATPase activity, thus coupling ATP hydrolysis with the formation of Fe-S clusters. The source of Fe is not yet known. The newly assembled Fe-S cluster is then transferred to a carrier protein, which delivers the Fe-S cluster to recipient apoproteins and converts recipient apoproteins (Apo) into holoproteins (Holo). Plastidial sulfur transferases include SufE1, SufE2, and SufE3. The Suf scaffold complex is composed of three different proteins: SufB, sulfur mobilization protein $\mathrm{C}$ (SufC), and sulfur mobilization protein $\mathrm{D}$ (SufD), primarily in a 1:2:1 $\left(\mathrm{BC}_{2} \mathrm{D}\right)$ ratio. Potential plastidial Fe-S carriers include sulfur mobilization protein $\mathrm{A} 1$ (SufA1), nitrogen fixation subunit U 1, 2, and 3 (NFU1, NFU2, and NFU3), high chlorophyll fluorescence 101 (HCF101), plastidial Clusters of Orthologous Groups 0354 protein (COG0354p), and glutaredoxin S14 and S16 (GRXS14 and GRXS16). Pi, inorganic phosphate.

system specifically deals with nitrogenase cofactor synthesis in nitrogen-fixing bacteria (Jacobson et al., 1989; Frazzon and Dean, 2003), the assembly of Fe-S clusters for other Fe-S proteins in bacteria is carried out by the iron-sulfur cluster (ISC) system and the sulfur mobilization (SUF) system (Takahashi and Tokumoto, 2002; Outten et al., 2004). The bacterial ISC system contains the following proteins: Cys desulfurase IscS, scaffold protein IscU, DnaK-like chaperone HscA (heat shock cognate protein A), DnaJ-like co-chaperone HscB (heat shock cognate protein B), and possibly Fd (Ayala-Castro et al., 2008; Roche et al., 2013). The bacterial SUF system contains two complexes: SufSE (where SufS is Cys desulfurase and SufE is sulfur transferase), and $\mathrm{SufBC}_{2} \mathrm{D}$ (the scaffold complex). The bacterial ISC and SUF systems have different tolerance to oxidative stress (Dai and Outten, 2012). In SufS, the catalytic active Cys residue is deeply buried and thus not easily accessed by $\mathrm{O}_{2}$ or $\mathrm{H}_{2} \mathrm{O}_{2}$ (Lima, 2002). On the contrary, the catalytic active Cys residue in IscS is exposed to the surface of the protein (Cupp-Vickery et al., 2003). Therefore, it was proposed that the ISC system is the housekeeping system for Fe-S assembly and the SUF system is adapted to assemble Fe$S$ clusters under Fe or S starvation, or oxidative stress (Outten et al., 2004). Consistent with this hypothesis, SufS was found to express under Fe starvation, low $\mathrm{S}$ availability, and oxidative stress conditions (Outten et al., 2004). If readers are interested in detailed information on the regulation of bacterial ISC and SUF systems and the functions of individual $\mathrm{Fe}-\mathrm{S}$ assembly proteins, they may refer to reviews on bacterial Fe-S assembly pathways, for example, Ayala-Castro et al. (2008) and Roche et al. (2013), and the references therein.

There are three Fe-S assembly and transfer pathways in the plant cell: the SUF pathway in the plastid, the ISC pathway in the mitochondrion, and the cytosolic iron-sulfur assembly (CIA) pathway (Balk and Pilon, 2011; Couturier et al., 2013; Balk and Schaedler, 2014). These pathways supply Fe-S clusters to the plastids, mitochondria, and cytosol plus nucleus, respectively. It is worth mentioning that the ISC pathway in plants evolved from the bacterial ISC system, which has poor tolerance to oxidative stress, and the SUF pathway in plants evolved from the bacterial SUF system, which has higher tolerance to oxidative stress (Takahashi and Tokumoto, 2002; Outten et al., 2004). Oxygenic phototrophs such as cyanobacteria and plants generate oxygen in photosynthetic cells or tissues. Therefore, it is likely that cyanobacteria and plants inherited the bacterial SUF system to tolerate the high oxidative environment at the site of photosynthesis. The plastidial SUF pathway appears to be independent from the mitochondrial ISC pathway and the CIA pathway (Van Hoewyk et al., 2007; Bernard et al., 2013). However, the CIA pathway requires the function of the mitochondrial ISC pathway: the sulfide compound from the ISC way is exported from the mitochondrion to the cytosol via ATM3 (․P binding cassette transporter of the mitochondrion 3) and it serves as the $\mathrm{S}$ source for the CIA pathway (Kushnir et al., 2001; Kim et al., 2006; Bernard et al., 2009). This review focuses on recent discoveries on the molecular functions of different types of proteins involved in the plastidial SUF pathway. Detailed information about the ISC and ICA pathways in plants could be found in the following excellent reviews: Balk and Pilon (2011), Couturier et al. (2013), and Balk and Schaedler (2014).

\section{Cys Desulfurase}

Cys desulfurase removes S from Cys and converts Cys to alanine (Ala) (Figure 2) (Zheng et al., 1993, 1994; Zheng and Dean, 1994). The newly released sulphane $S\left(S^{0}\right)$ is bound to the active site of Cys desulfurase in the form of persulfide (R-S-S $\left.{ }^{0} H\right)$. The bound sulphane $\left(\mathrm{S}^{0}\right)$ is then transferred in the same form to the $\mathrm{Fe}-\mathrm{S}$ scaffold complex with the help from sulfur transferase.

Cys desulfurases can be classified into two groups according to their sequences and structures (Mihara et al., 1997; Mihara and Esaki, 2002; Roret et al., 2014). The two groups differ in the $\beta$-hairpin loop and the extended lobe containing the catalytic Cys residue. In group I, the extended loop lacks the $\beta$-hairpin structure and it is long and sufficiently flexible to transfer $\mathrm{S}^{2-}$ to sulfur transferase (Kaiser et al., 2000). Thus the sulfur transferase 
associated with a group I Cys desulfurase does not need to be flexible to acquire $\mathrm{S}^{2-}$. On the contrary, group II Cys desulfurases have a $\beta$-hairpin structure constraining the catalytic site and their extended loop is short and insufficiently flexible (Mihara et al., 2002; Outten et al., 2003; Singh et al., 2013). Therefore, the sulfur transferase associated with a group II Cys desulfurase needs additional flexibility to acquire $S^{2-}$ (Kim and Park, 2013).

Plants have three Cys desulfurases: nitrogen fixation Slike 1 (NFS1/NifS1, referred to as NFS1 hereafter) (Frazzon et al., 2007), nitrogen fixation S-like 2/chloroplastic nitrogen fixation S-like/chloroplastic sulfur mobilization protein $\mathrm{S}$ (NFS2/CpNifS/CpSufS, referred to as NFS2 hereafter) (PilonSmits et al., 2002; Ye et al., 2005; Van Hoewyk et al., 2007), and abscisic acid deficient 3 (ABA3) (Heidenreich et al., 2005). Among them, ABA3 is involved in Mo cofactor sulfuration but not in Fe-S assembly (Bernard et al., 2013). NFS1 is a group I Cys desulfurase (Mihara et al., 1997; Mihara and Esaki, 2002) and it is targeted to the mitochondrion (Kushnir et al., 2001). NFS2 is a group II Cys desulfurase (Mihara et al., 1997; Mihara and Esaki, 2002) and it is located to the chloroplast (Table 1) (Léon et al., 2002; Pilon-Smits et al., 2002). Full-length NFS2 has a 35-amino acid plastid transit peptide and a C-terminal Cys desulfurase domain (Figure 3) (Pilon-Smits et al., 2002). Gel filtration of the purified NFS2 protein showed this protein forms a dimer (Table 2) (Pilon-Smits et al., 2002; Ye et al., 2005). Recombinant NFS2 demonstrated Cys desulfurase activity toward Cys and seleno-Cys lyase activity toward seleno-Cys, with a much high activity on seleno-Cys (Pilon-Smits et al., 2002). Absorption spectrum indicated the presence of a pyridoxal $5^{\prime}$-phosphate cofactor in the purified protein (Pilon-Smits et al., 2002). NFS2 silencing via RNA interference (RNAi) is lethal (Van Hoewyk et al., 2007). Immunoblot analysis showed that the levels of Fe-S proteins representing different types of plastidial $\mathrm{Fe}-\mathrm{S}$ clusters were reduced in the RNAi lines. However, mitochondrial Fe-S proteins and respiration were not affected in the RNAi lines. Taken together, these data indicate that NFS2 is required in de novo assembly of all plastidial Fe-S clusters and that the plastidial and mitochondrial $\mathrm{Fe}-\mathrm{S}$ assembly pathways operate independently (Van Hoewyk et al., 2007).

\section{Sulfur Transferase}

The Cys desulfurase activity of NFS2 is activated by sulfur transferases, which accept the persulfide (R-S-S $\left.{ }^{0} \mathrm{H}\right)$ from NFS2 and transfer it to the Fe-S scaffold complex (Figure 2). Plants have three sulfur transferases: sulfur mobilization protein E1, E2, and E3, i.e., SufE1, SufE2, and SufE3 (Table 1) (Xu and Møller, 2006; Ye et al., 2006; Murthy et al., 2007). Fulllength SufE1 contains a transit peptide, a SufE domain, and a BolA domain (Figure 3) (Xu and Møller, 2006; Ye et al., 2006). In silico analysis of the transit peptide suggests that SufE1 is dual-targeted to plastids and mitochondria. This prediction was confirmed with confocal microscopic analysis of tobacco and Arabidopsis leaves transiently expressing the AtSufE1-YFP (At stands for A. thaliana; YFP stands for yellow fluorescent protein) fusion protein (Xu and Møller, 2006). AtSufE1 was able to complement the growth defects of $\Delta$ SufE Escherichia coli. In vitro Cys desulfurization assays demonstrated that AtSufE1 interacts with and activates both plastid-targeted NFS2 and mitochondrion-targeted NFS1 (Table 2) (Xu and Møller, 2006; Ye et al., 2006). Loss-of-function AtSufE1 mutants are embryo lethal, indicating that SufE1 and Fe-S cluster assembly in plastids and mitochondria are essential during embryo development (Xu and Møller, 2006; Ye et al., 2006).

The function of the BolA domain in SufE1 was intriguing. BolA proteins are known as morphogens, whose overexpression results in spherical cell morphology in E. coli (Aldea et al., 1988). Surprisingly, overexpression of the BolA domain of AtSufE1 showed no effects on the morphology of E. coli or Arabidopsis (Xu and Møller, 2006). Therefore, Xu and Møller (2006) proposed that the BolA domain in SufE1 is not functional. However, recent yeast-two-hybrid and bimolecular fluorescence complementation assays demonstrated that the BolA domain allows SufE1 to interact with monothiol glutaredoxins (GRXs), including plastid-targeted GRXS14 and GRXS16 (Couturier et al., 2014). In addition, in vitro experiments showed that the binding of GRXs promotes the deglutathionation of SufE1, and thereby facilitates the activation of NFS2 by deglutathionated SufE1 (Couturier et al., 2014). Taken together, the interactions between the SufE1 BolA domain and GRXs suggest possible redox regulation of SufE1 activity and $\mathrm{Fe}-\mathrm{S}$ cluster assembly by GRXs.

Full-length SufE2 and SufE3 have a plastid transit peptide and a SufE domain (Figure 3). Confocal microscopic analysis of Arabidopsis protoplasts expressing GFP (green fluorescent protein)-tagged AtSufE2 and AtSufE3 confirmed their plastidial localization (Murthy et al., 2007). The SufE domain of AtSufE2 and AtSufE3 was found to interact with and activate the Cys desulfurase activity of NFS2 (Table 2) (Murthy et al., 2007). SufE3 has a C-terminal NadA domain. In bacteria, the NadA gene encodes quinolinate synthase A (EC 2.5.1.72), an enzyme required for NAD biosynthesis (Foster and Moat, 1980). Expression of AtSufE3 complemented the E. coli $\triangle \mathrm{NadA}$ mutant, suggesting that SufE3 contains a functional NadA domain (Murthy et al., 2007). Consistent with this hypothesis, recombinant AtSufE3 demonstrated quinolinate synthase (QS) activity. The expression of the NadA domain of AtSufE3 alone could not complement the E. coli $\triangle \mathrm{NadA}$ mutant, indicating that the SufE domain of SufE3 is required for QS activity. Apparently, QS activity requires the $4 \mathrm{Fe}-4 \mathrm{~S}$ cluster in the NadA domain; and the SufE domain is responsible for reconstituting the cluster by interacting with NFS2 (Murthy et al., 2007).

The phenotype of loss-of-function AtSufE2 mutants has not yet been reported. Loss-of-function AtSufE3 mutants are embryo lethal (Murthy et al., 2007), suggesting that SufE3 is an essential protein. This is somewhat surprising because there are three SufE proteins in plants. It is possible that the BolA domain in SufE1 and the NadA domain in SufE3 make the two proteins irreplaceable (Murthy et al., 2007). Differential expression could be another reason why the three SufE proteins cannot complement each other (Murthy et al., 2007). For example, SufE1 and SufE3 are expressed in vegetative tissues while SufE2 is only expressed in pollen. Furthermore, SufE1 is 


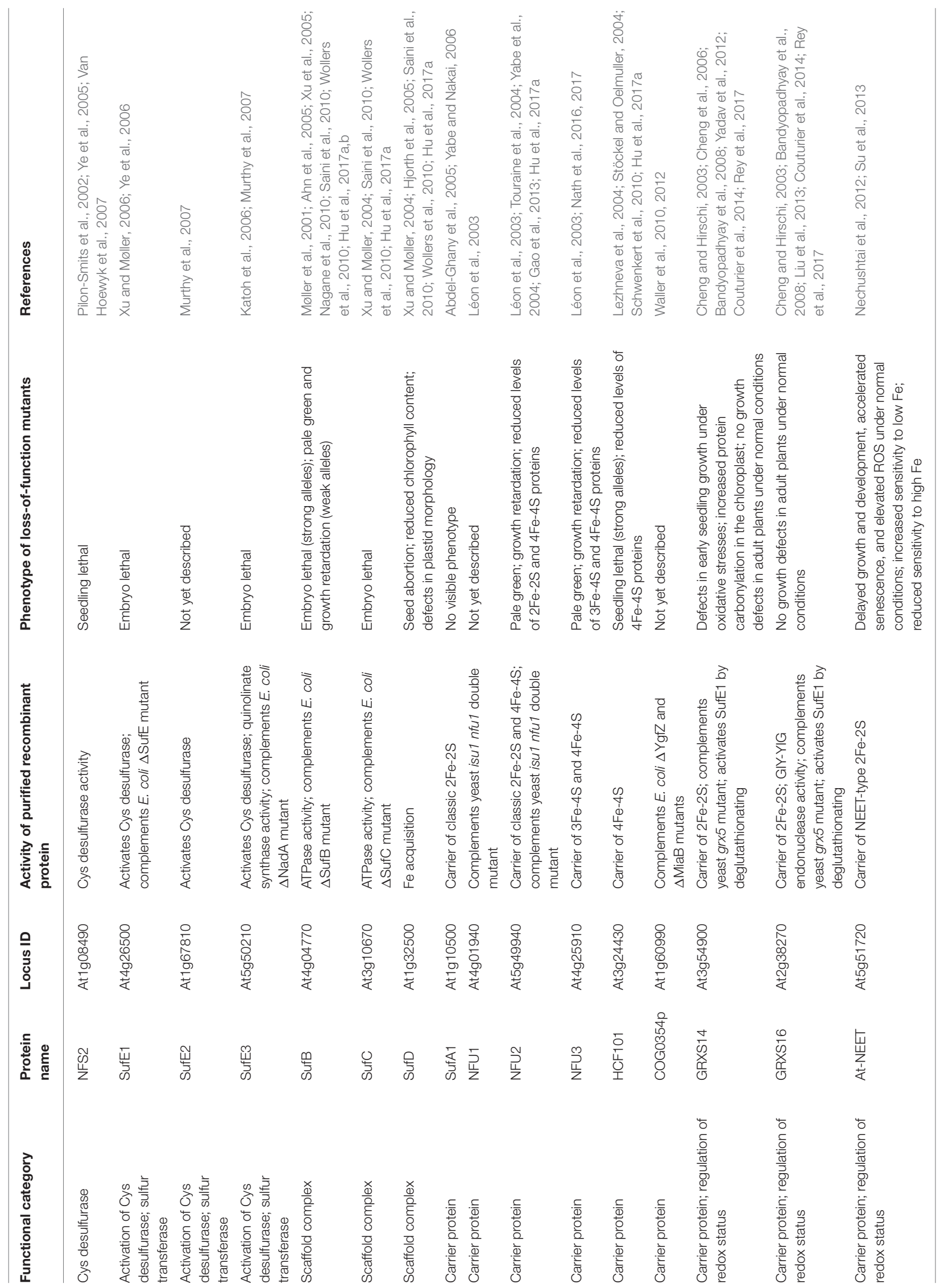




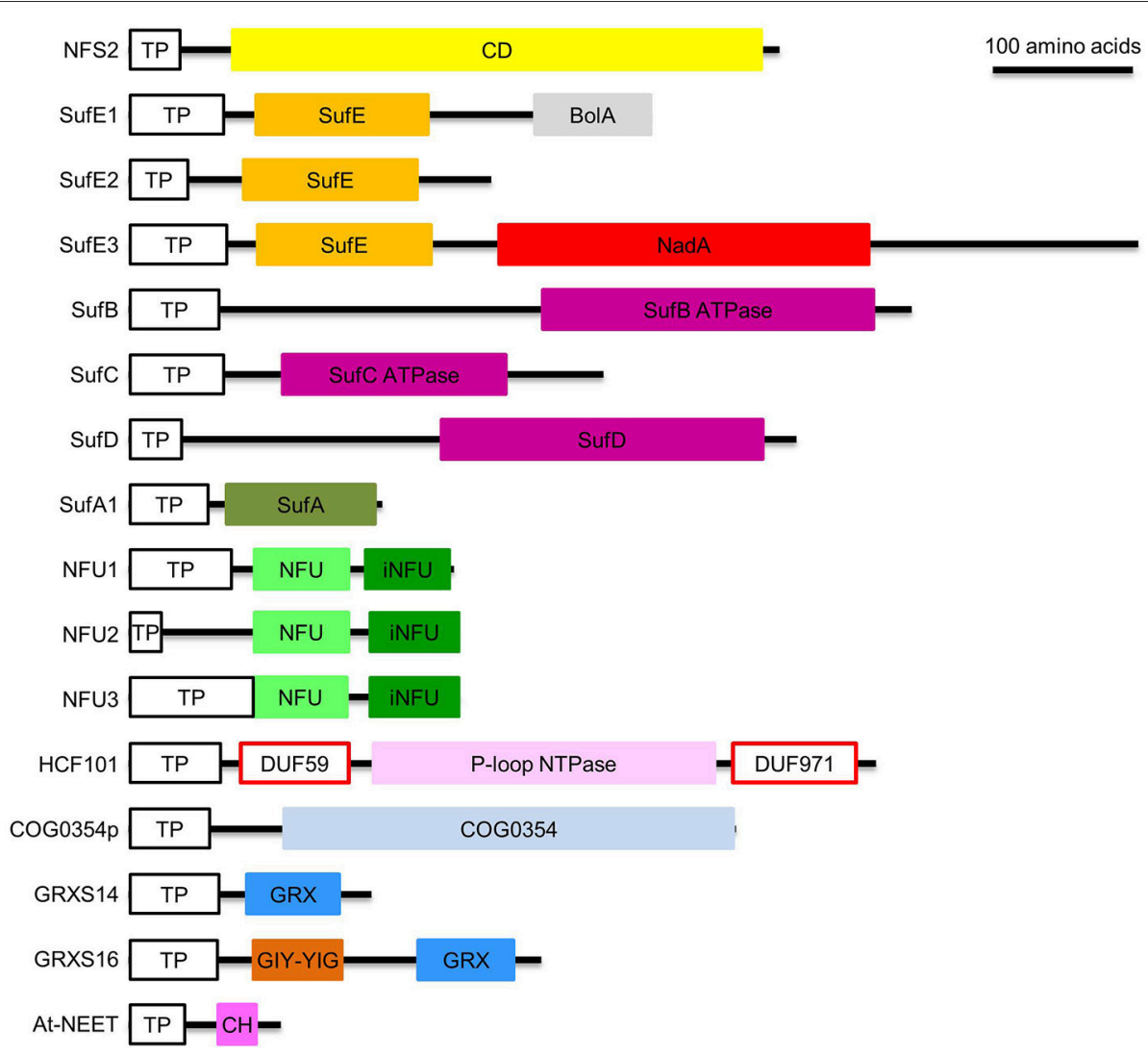

FIGURE 3 | Domain composition of proteins involved in de novo assembly and transfer of Fe-S clusters in the plastid. ATPase, adenosine triphosphatase; BolA, DNA-binding transcriptional regulator BolA; CD, Cys desulfurase; CH, CDGSH motif; COG0354, Clusters of Orthologous Groups 0354 protein; COG0354p, plastidial Clusters of Orthologous Groups 0354 protein; DUF59 and DUF971; domain of unknown function 59 and 971; GIY-YIG, GlylleTyr-TyrlleGly; GRX, glutaredoxin; GRXS14 and GRXS16, glutaredoxin S14 and S16; HCF101, high chlorophyll fluorescence 101; iNFU, redox-inactive nitrogen fixation subunit U; NadA, quinolinate synthase A; NFU, nitrogen fixation subunit U; NFU1, NFU2, and NFU3, nitrogen fixation subunit U 1, 2, and 3; NFS2, nitrogen fixation S-like 2; P-loop NTPase, P-loop nucleotide phosphatase; SufA1, SufB, SufC, SufD, SufE1, SufE2, and SufE3, sulfur mobilization protein A1, B, C, D, E1, E2, and E3; TP, transit peptide. Note that SufE1 and At-NEET are dually targeted to plastids and mitochondria. Bar $=100$ amino acids.

dual-targeted to both plastids and mitochondria while SufE2 and SufE3 are targeted to the plastid.

\section{The Fe-S Scaffold Complex}

Persulfide (R-S-S $\left.{ }^{0} \mathrm{H}\right)$ groups are transferred to the scaffold complex for Fe-S cluster assembly (Figure 2). During this step, sulphane $\left(S^{0}\right)$ accepts electrons from electron donors and is reduced to sulfide $\left(\mathrm{S}^{2-}\right)$, the final form of $\mathrm{S}$ in Fe-S clusters. The scaffold complex of the SUF pathway is composed of three different proteins: sulfur mobilization protein $\mathrm{B} /$ non-intrinsic $A B C$ protein 1 (SufB/NAP1; referred to as SufB hereafter; $A B C$ stands for ATP binding cassette), sulfur mobilization protein C/non-intrinsic ABC protein 7 (SufC/NAP7; referred to as SufC hereafter), and sulfur mobilization protein $\mathrm{D} /$ non-intrinsic $\mathrm{ABC}$ protein 6 (SufD/NAP6; referred to as SufD hereafter), primarily in a 1:2:1 $\left(\mathrm{BC}_{2} \mathrm{D}\right)$ ratio (Xu et al., 2005; Hu et al., 2017a). However, other subcomplexes may form as well (Wollers et al., 2010; Roche et al., 2013; Hu et al., 2017a). Extensive proteinprotein interactions have been observed among SufB, SufC, and SubD proteins (Table 2) (Xu and Møller, 2004; Xu et al., 2005;
Hu et al., 2017a). Although all three proteins are required for in vivo assembly of Fe-S clusters (Table 1), formation of Fe-S clusters occurs directly on SufB (Saini et al., 2010). Each SufBC ${ }_{2}$ D complex binds to one molecule of $\mathrm{FADH}_{2}$ via SufB (Wollers et al., 2010). $\mathrm{FADH}_{2}$ was thought to provide electrons to reduce sulphane $\left(S^{0}\right)$ to sulfide $\left(S^{2-}\right)$ (Wollers et al., 2010). However, Wollers et al. (2010) reported that $\mathrm{FADH}_{2}$ actually plays a role in reductive mobilization of $\mathrm{Fe}$, which is equally important for the assembly of Fe-S clusters. Using lysed and intact chloroplasts, Takahashi et al. showed that plastidial formation of Fe-S clusters on the scaffold complex requires ATP and NADPH (Takahashi et al., 1986, 1991a,b). These observations were re-confirmed by the activation of the SUF pathway in cell lysates by the addition of ATP and NADPH (Saini et al., 2010). The hydrolysis of ATP is likely carried out by the $\mathrm{SufBC}_{2} \mathrm{D}$ complex, because the SufB and SufC subunits of this complex have ATPase activity (Xu and Møller, 2004; Xu et al., 2005). While ATP hydrolysis by the $\mathrm{SufBC}_{2} \mathrm{D}$ complex provides energy for the formation of Fe-S clusters (Xu and Møller, 2004; Xu et al., 2005), NADPH may serve as the reducing agent for redox cycling of $\mathrm{FADH}_{2} / \mathrm{FAD}$ (Saini 
TABLE 2 | Protein-protein interactions among different plastidial Fe-S assembly and transfer factors.

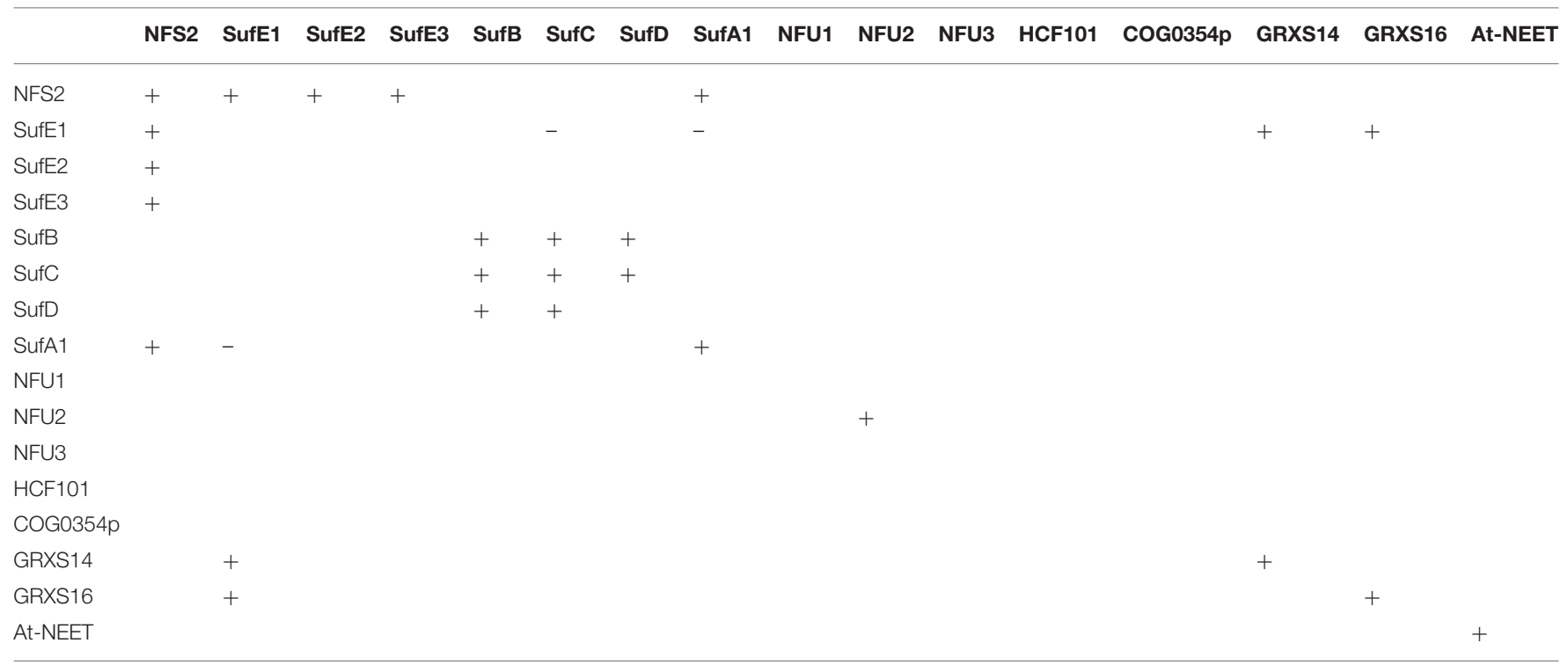

et al., 2010). In addition to NADPH, another possible source of reducing agents is plant-type Fds. Because plant-type Fds participate in many plastidial reactions, direct evidence for their role in the SUF pathway is still lacking. However, mitochondrial $\mathrm{Fd}$ has been shown to be essential for de novo assembly of Fe-S clusters in yeast mitochondria (Takahashi and Nakamura, 1999).

The source of Fe for plastidial Fe-S assembly is not yet known. Universal $\mathrm{Fe}$-storage protein ferritin was previously considered as a candidate to supply $\mathrm{Fe}$ to the $\mathrm{Fe}-\mathrm{S}$ scaffold complex (Lobreaux and Briat, 1991; Briat and Lobréaux, 1997). However, the triple loss-of-function Arabidopsis mutant of three major chloroplastic ferritins FER1, FER3, and FER4 had normal photosynthetic apparatus, which is extremely dependent on proper assembly of Fe-S clusters (Ravet et al., 2009). These results suggest that chloroplastic ferritins do not constitute a major Fe pool for the plastidial SUF pathway. Interestingly, the triple mutant exhibited increased sensitivity to excess Fe, suggesting that chloroplastic ferritins may actually act as Fe scavengers.

Full-length SufB contains a plastid transit peptide and an adenosine triphosphatase (ATPase, EC 3.6.1.3) domain with degenerative Walker A (also known as P-loop) and Walker B motifs (Figure 3) (Xu et al., 2005). The plastidial localization of AtSufB was confirmed with confocal microscopic analysis of onion epidermal cells expressing the AtSufB-GFP fusion protein (Møller et al., 2001). AtSufB expression complemented the growth defects of $E$. coli $\Delta$ SufB mutant, suggesting that AtSufB is a functional SufB protein (Xu et al., 2005). However, the absence of either SufD or both SufC and SufD caused substantial reduction in the in vivo cluster-assembly ability of SufB (Hu et al., 2017a). These results indicate that SufC and SufD are required for in vivo Fe-S cluster formation on SufB. Recombinant AtSufB demonstrated $\mathrm{Fe}^{2+}$-stimulated ATPase activity (Xu et al., 2005). This is somewhat surprising because ATPase activity was not reported for prokaryotic SufB (Roche et al., 2013). SufB was previously classified as a non-intrinsic
$\mathrm{ABC}$ protein; however its ATPase domain does not contain the conserved $\mathrm{ABC}$ signature motif. In addition, SufB is capable of forming homodimers (Table 2), which also is different from its prokaryotic counterparts. The strong loss-of-function AtSufB mutant is embryo lethal, suggesting that SufB and the SUF pathway are essential to embryogenesis (Nagane et al., 2010). Weak SufB alleles in Arabidopsis and Nicotiana benthamiana had pale green leaves and retarded growth, due to defects in chlorophyll biosynthesis and chloroplast development (Møller et al., 2001; Ahn et al., 2005; Nagane et al., 2010; Hu et al., $2017 a, b)$. These data demonstrate that insufficient supply of Fe-S clusters in the plastid will lead to defects in chlorophyll biosynthesis, chloroplast development, growth retardation, and embryo development.

Full-length SufC contains a plastid transit peptide and an ATPase domain with degenerative Walker A and Walker B motifs and an ABC signature motif (Figure 3) (Xu and Møller, 2004). The plastidial localization of SufC was confirmed by microscopic analysis of tobacco leaves expressing the AtSufCYFP fusion protein (Xu and Møller, 2004). AtSufC expression complemented the growth defects of $E$. coli $\triangle$ SufC mutant, suggesting that AtSufC is a functional SufC protein (Xu and Møller, 2004). Consistent with this observation, recombinant AtSufC demonstrated $\mathrm{Mg}^{2+}$-stimulated ATPase activity (Xu and Møller, 2004). Loss of AtSufC resulted in an embryo-lethal phenotype, suggesting that SufC and the SUF pathway are essential to embryogenesis (Xu and Møller, 2004). The RNAi lines of SufB demonstrated pale green leaves, because of their defects in chlorophyll biosynthesis (Hu et al., 2017a). Due to similarity to the ISC pathway, it was proposed that ATP hydrolysis by SufB and SufC induces conformational changes of the $\mathrm{SufBC}_{2} \mathrm{D}$ scaffold complex to release newly assembled $\mathrm{Fe}$ S clusters (Markley et al., 2013). However, Saini et al. (2010) reported that the ATPase activity is instead necessary for $\mathrm{Fe}$ acquisition during in vivo assembly of Fe-S clusters. 
Full-length SufD contains a plastid transit peptide and a SufD domain (Figure 3). The plastidial localization of SufD was confirmed by microscopic analysis of tobacco leaves expressing the AtSufD-YFP fusion protein (Xu and Møller, 2004). Unlike SufB or SufC, the SufD domain does not contain Walker A, Walker B, or the ABC signature motif. Saini et al. (2010) reported that SufD is not required for in vivo sulfur transfer from the NFS2-SufE system, but it is required during in vivo Fe acquisition and in vivo formation of Fe-S clusters on SufB (Saini et al., 2010). The loss-of-function mutation in the SufD gene resulted in seed abortion, reduced chlorophyll content, and defects in plastid morphology (Hjorth et al., 2005). Consistent with these observations, RNAi lines of this protein showed defective chlorophyll biosynthesis (Hu et al., 2017a). Taken together, these data indicate that SufD may have a general house-keeping role (e.g., chlorophyll biosynthesis and chloroplast development), instead of functioning only in embryogenesis (Hjorth et al., 2005).

\section{Fe-S Carrier Proteins}

$\mathrm{Fe}-\mathrm{S}$ clusters assembled on the $\mathrm{SufBC}_{2} \mathrm{D}$ scaffold complex are transferred to recipient proteins via carrier proteins (Figure 2). When exogenous $\mathrm{Fe}^{2+}$ and $\mathrm{S}^{2-}$ are supplied, Fe-S carrier proteins may serve as scaffold proteins for in vitro $\mathrm{Fe}-\mathrm{S}$ cluster assembly. Examples of plastidial Fe-S carrier proteins include: sulfur mobilization protein A1/chloroplast ISC protein A1 (SufA1/CpIscA1, referred to as SufA1 hereafter) (Abdel-Ghany et al., 2005; Yabe and Nakai, 2006); nitrogen-fixation-subunitU-type proteins NFU1, NFU2, and NFU3 (Léon et al., 2003; Touraine et al., 2004; Yabe et al., 2004; Gao et al., 2013; Nath et al., 2016, 2017); P-loop nucleotide phosphatase (NTPase) HCF101 (high chlorophyll fluorescence 101) (Lezhneva et al., 2004; Stöckel and Oelmuller, 2004; Schwenkert et al., 2010); plastidial-type COG0354 (COG0354p, COG stands for Clusters of Orthologous Groups; p stands for plastidial) (Waller et al., 2010, 2012); and monothiol GRXS14 and GRXS16 (S refers to the Ser residue in the CGFS active site motif of GRXs) (Table 1) (Cheng and Hirschi, 2003; Cheng et al., 2006; Bandyopadhyay et al., 2008; Yadav et al., 2012; Couturier et al., 2014).

\section{SufA1}

The Arabidopsis genome encodes four SufA proteins: SufA1 is plastid-targeted (Figure 3; Table 1) while SufA2, SufA3, and SufA4 are mitochondrion-targeted (Yabe and Nakai, 2006). All four SufA proteins contain three conserved Cys residues: one in the GCXGXXY motif and the other two in the Cterminal C(G/S)CXSF motif (Yabe and Nakai, 2006). These conserved Cys residues may serve as ligands for Fe-S clusters. The plastidial localization of SufA1 was confirmed with a number of independent techniques, including confocal microscopic analysis of GFP-fusion protein, import assays, as well as chloroplast sub-fractionation and subsequent immunodetection (AbdelGhany et al., 2005). SufA1 is ubiquitously expressed in all the Arabidopsis tissues tested, with higher expression in green tissues such as leaves and flower stalks (Abdel-Ghany et al., 2005; Yabe and Nakai, 2006). Gel filtration analysis showed that SufA1 tends to form homodimers (Table 2). Recombinant SufA1 was capable of enhancing NFS2-mediated in vitro assembly of $2 \mathrm{Fe}-2 \mathrm{~S}$ clusters on apo Fd (Abdel-Ghany et al., 2005). This observation suggests a possible interaction between SufA1 and NFS2 (Table 2), during which SufA1 may act as a scaffold protein for in vitro assembly of $2 \mathrm{Fe}-2 \mathrm{~S}$ clusters. However, loss-of-function mutation in the SufA1 gene does not cause any defect in plant growth and development or in the abundance of classic $2 \mathrm{Fe}-2 \mathrm{~S}$ protein $\mathrm{Fd}$ (Yabe and Nakai, 2006). It is likely that SufA1 serves as a back-up carrier for classic $2 \mathrm{Fe}-2 \mathrm{~S}$ (Figure 4A), when other classic 2Fe-2S carriers are insufficient, e.g., under stress conditions.

\section{NFU1, NFU2, and NFU3}

The Arabidopsis genome encodes five NFU proteins: NFU1, NFU2, and NFU3 are plastid-localized (Figure 3; Table 1) and NFU4 and NFU5 are mitochondrion-localized (Léon et al., 2003; Yabe et al., 2004; Gao et al., 2013; Nath et al., 2016). Their subcellular localization was confirmed with confocal microscopic analysis of Arabidopsis protoplasts expressing GFP-tagged NFU proteins (Léon et al., 2003). Reverse transcription-PCR analysis showed that NFU1 is expressed in flower stalks and siliques, NFU2 is expressed in flower stalks and leaves, and NFU3 is expressed in flower stalks, leaves, flowers, and roots (Léon et al., 2003).

Mature NFU1, NFU2, and NFU3 contain an N-terminal redox-active NFU domain with a conserved CXXC motif and a C-terminal redox-inactive NFU domain (Léon et al., 2003; Gao et al., 2013; Nath et al., 2016). The two Cys residues in the conserved CXXC motif of the redox-active NFU domain are used to coordinate Fe-S clusters. Both NFU1 and NFU2 were able to complement the growth defects of the yeast isu1 nful double mutant, suggesting that NFU1 and NFU2 are functional NFU proteins (Léon et al., 2003). The oligomerization and activity of recombinant NFU1 has not yet been tested in vitro. Recombinant NFU2 may form homodimers and homotetramers (Table 2) (Yabe et al., 2004; Gao et al., 2013). It served as a scaffold protein during in vitro reconstitution of classic $2 \mathrm{Fe}-2 \mathrm{~S}$ and $4 \mathrm{Fe}-4 \mathrm{~S}$ clusters (Figure $4 \mathrm{~A}$ ) and a carrier protein during subsequent in vitro transfer to apo GRXS16 and APR1 (adenosine 5 '-phosphosulfate reductase 1, EC 1.8.99.2) proteins (Léon et al., 2003; Yabe et al., 2004; Gao et al., 2013). The unidirectional and intact transfer of classic $2 \mathrm{Fe}-2 \mathrm{~S}$ from NFU2 to apo GRXS16 in vitro suggests that NFU2 may work upstream of GRXS16 during in vivo delivery of classic $2 \mathrm{Fe}-2 \mathrm{~S}$ clusters to recipient proteins (Figure 4C) (Gao et al., 2013). NFU3 also is a functional NFU protein. Recombinant NFU3 served as a scaffold protein during in vitro reconstitution of $3 \mathrm{Fe}-4 \mathrm{~S}$ and $4 \mathrm{Fe}-4 \mathrm{~S}$ clusters (Figure 4A) (Nath et al., 2016).

The phenotype of loss-of-function mutants of NFU1 has not been described. Therefore, it is not clear which Fe-S cluster(s) NFU1 is able to carry. Loss-of-function mutations of the NFU2 gene resulted in smaller and pale green leaves, a smaller plant size, as well as stunted growth and development (Touraine et al., 2004; Yabe et al., 2004). SDS-PAGE and immunoblot analysis showed that the levels of classic $2 \mathrm{Fe}-2 \mathrm{~S}$ protein $\mathrm{Fd}, 4 \mathrm{Fe}-4 \mathrm{~S}$ proteins $\mathrm{PsaA}, \mathrm{PsaB}$, and $\mathrm{PsaC}$, and siroheme $4 \mathrm{Fe}-4 \mathrm{~S}$ proteins $\mathrm{NiR}$ and $\mathrm{SiR}$ were largely reduced in the $n f u 2$ mutants while the level of Rieske-type $2 \mathrm{Fe}-2 \mathrm{~S}$ protein PetC was substantially 


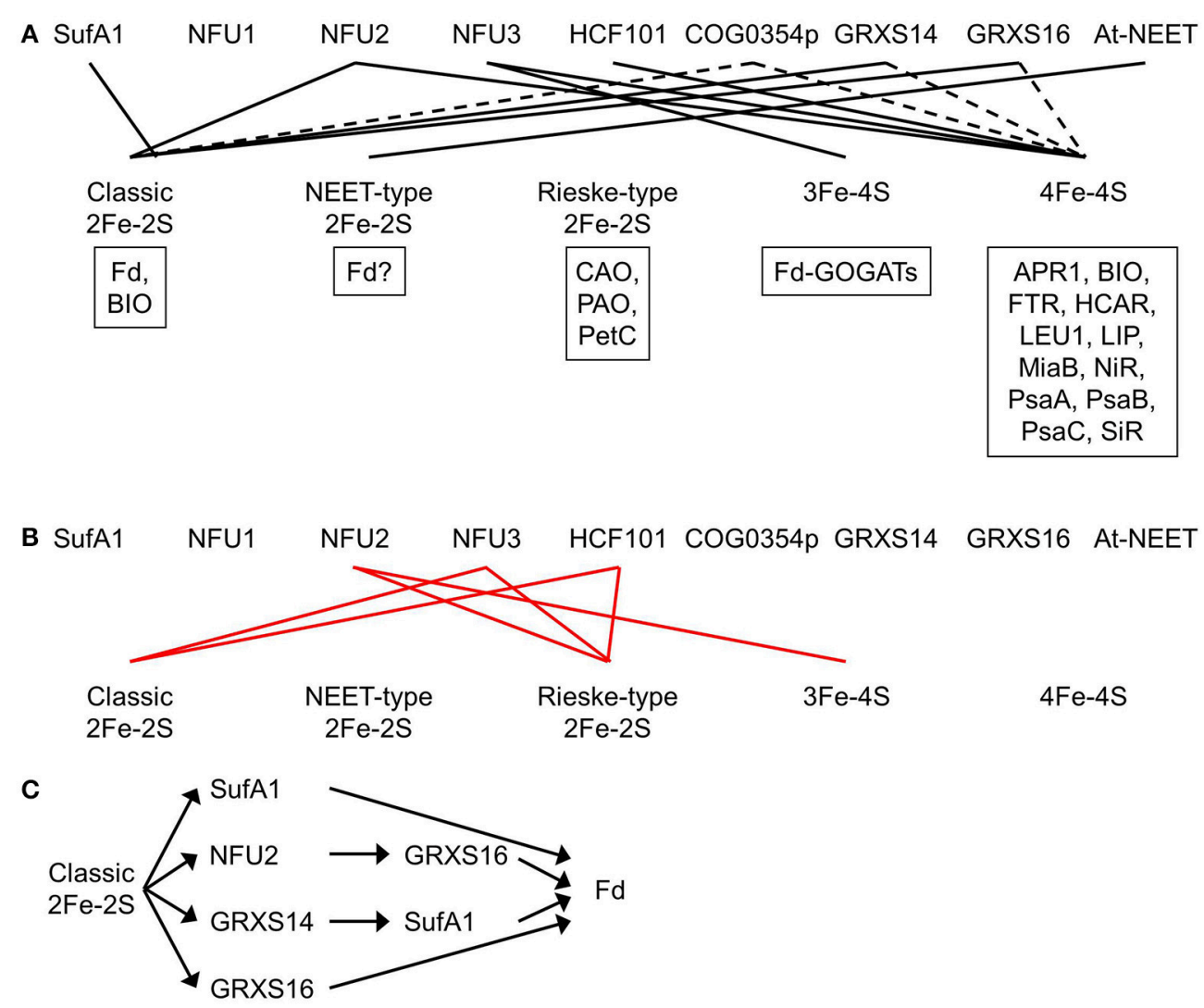

FIGURE 4 | Plastidial Fe-S clusters and the corresponding Fe-S carrier proteins. (A) Plastidial Fe-S clusters, the corresponding Fe-S carrier proteins, and exemplar Fe-S proteins. Solid black lines indicate that the Fe-S carrier protein has been shown to act as a carrier for that type of Fe-S clusters. Dashed lines indicate that the Fe-S carrier protein could be a possible carrier for that type of Fe-S clusters. Exemplar Fe-S proteins containing that type of Fe-S clusters are shown in boxes. APR1, adenosine 5'-phosphosulfate reductase 1; At-NEET, Arabidopsis thaliana NEET; BIO, biotin synthase; CAO, chlorophyllide a oxygenase; COG0354p, plastidial Clusters of Orthologous Groups 0354 protein; Fd, ferredoxin; Fd-GOGATs, ferredoxin-dependent Gln oxoglutarate aminotransferases; FTR, ferredoxin-thioredoxin reductase; GRXS14 and GRXS16, glutaredoxin S14 and S16; HCAR, 7-hydroxymethyl chlorophyll a reductase; HCF101, high chlorophyll fluorescence 101; LEU1, 3-isopropylmalate isomerase 1; LIP, lipoic acid synthase; MiaB, isopentenyl-adenosine A37 tRNA methylthiolase; NFU1, NFU2, and NFU3, nitrogen fixation subunit U 1, 2, and 3; NiR, nitrite reductase; PetC, photosynthetic electron transfer C; PAO, pheophorbide a oxygenase; PsaA, PsaB, and PsaC, Photosystem I proteins A, B, and C; SiR, sulfite reductase. Note that BIO contains one classic 2Fe-2S and one 4Fe-4S. Recombinant At-NEET was able to transfer the NEET-type 2Fe-2S cluster to the plant-type Fd under in vitro conditions. However, it is not clear whether the plant-type Fd is able to serve as a recipient protein for the NEET-type $2 \mathrm{Fe}-2 \mathrm{~S}$ cluster under in vivo conditions, because plant-type Fds are known as classic 2Fe-2S proteins. Therefore, a question mark is placed next to Fd in the box for exemplar NEET-type 2Fe-2S proteins. (B) Experimental evidence showed that NFU2 does not act as a carrier for Rieske-type 2Fe-2S and 3Fe-4S cluster and that NFU3 and HCF101 do not act as carriers for classic and Rieske-type 2Fe-2S clusters. Solid red lines indicate that the Fe-S carrier protein does not transfer that type of Fe-S clusters. (C) The transfer network of carrier proteins for classic 2Fe-2S clusters in the plastid. Fd is used as an example of recipient proteins.

increased. Consistent with the immunoblot data, the activities of PSI and SiR were substantially reduced in the $n f u 2$ mutants while the activity of $3 \mathrm{Fe}-4 \mathrm{~S}$ enzyme Fd-GOGAT was significantly increased. Taken together, these data demonstrate that NFU2 is required in the assembly and transfer of classic $2 \mathrm{Fe}-2 \mathrm{~S}$ and $4 \mathrm{Fe}-4 \mathrm{~S}$ clusters (Figure 4A). Excess Fe and $\mathrm{S}$ in the $n f u 2$ mutants could be used to make other $\mathrm{Fe}$-S clusters such as Rieske-type $2 \mathrm{Fe}-2 \mathrm{~S}$ and $3 \mathrm{Fe}-4 \mathrm{~S}$, hence, increased levels of Rieske-type $2 \mathrm{Fe}-2 \mathrm{~S}$ protein PetC and $3 \mathrm{Fe}-4 \mathrm{~S}$ proteins Fd-GOGATs. The elevated contents of PetC and Fd-GOGATs suggest that NFU2 does not serve as a carrier protein for Rieske-type $2 \mathrm{Fe}-2 \mathrm{~S}$ or $3 \mathrm{Fe}-4 \mathrm{~S}$ (Figure $4 \mathrm{~B}$ ).

Loss-of-function mutants of NFU3 exhibited retarded growth and development, along with smaller and pale green leaves and a smaller plant size (Nath et al., 2016, 2017). SDS-PAGE and immunoblot analysis showed that the levels of $4 \mathrm{Fe}-4 \mathrm{~S}$ proteins $\mathrm{PsaA}, \mathrm{PsaB}$, and $\mathrm{PsaC}$ and $3 \mathrm{Fe}-4 \mathrm{~S}$ proteins $\mathrm{Fd}-\mathrm{GOGATs}$ were substantially reduced in the nfu3 mutants while the levels of classic $2 \mathrm{Fe}-2 \mathrm{~S}$ protein $\mathrm{Fd}$ and Rieske-type $2 \mathrm{Fe}-2 \mathrm{~S}$ protein $\mathrm{PetC}$ were significantly increased. Consistent with the immunoblot data, PSI activity was nearly abolished in the $n f u 3$ mutants. Taken together, these results demonstrate that NFU3 is required in the assembly and transfer of $4 \mathrm{Fe}-4 \mathrm{~S}$ and $3 \mathrm{Fe}-4 \mathrm{~S}$ clusters (Figure $4 \mathrm{~A}$ ). Excess $\mathrm{Fe}$ and $\mathrm{S}$ in the $n f u 3$ mutants could be used to make other Fe-S clusters such as classic and Rieske-type 2Fe-2S, hence, increased levels of classic $2 \mathrm{Fe}-2 \mathrm{~S}$ protein $\mathrm{Fd}$ and Rieske-type $2 \mathrm{Fe}-$ $2 S$ protein PetC. The elevated levels of Fd and PetC suggest that NFU3 does not serve as a carrier protein for classic or Rieske-type 2Fe-2S (Figure 4B). 


\section{HCF101}

HCF101 is a plastid-localized P-loop NTPase with an Nterminal domain of unknown function 59 (DUF59) and a Cterminal domain of unknown function 971 (DUF971) (Figure 3; Table 1) (Lezhneva et al., 2004; Stöckel and Oelmuller, 2004; Schwenkert et al., 2010). The P-loop NTPase domain contains a Walker A motif (i.e., P-loop, CKGGVGKS), an A' motif (GARVGIFDADV), and a B motif (DYLVID) (Stöckel and Oelmuller, 2004; Schwenkert et al., 2010). Via site-directed mutagenesis, three Cys residues were found to be required for the assembly and/or stability of Fe-S clusters: $C^{128}, C^{347}$, and $C^{419}$ (Schwenkert et al., 2010). $C^{347}$ and $C^{419}$ exist in the presence of a second Cys residue: $\mathrm{C}^{339} \mathrm{X}_{7} \mathrm{C}^{347}$ and $\mathrm{C}^{414} \mathrm{X}_{4} \mathrm{C}^{419}$. These Cys residues and motifs might be binding sites for $\mathrm{Fe}-\mathrm{S}$ clusters. Analysis of $\mathrm{Fe}$ and $\mathrm{S}$ contents in reconstituted HCF101 showed that this protein may bind to one $4 \mathrm{Fe}-4 \mathrm{~S}$ cluster per monomer (Schwenkert et al., 2010).

The plastidial localization of HCF101 was confirmed with import assays (Lezhneva et al., 2004). Recombinant HCF101 served as a scaffold protein during in vitro reconstitution of $4 \mathrm{Fe}-4 \mathrm{~S}$ clusters and a carrier protein during subsequent in vitro transfer to apo LEU1 (3-isopropylmalate isomerase 1, EC 4.2.1.33) (Schwenkert et al., 2010). Complete loss-of-function mutations in the HCF101 gene resulted in a seedling-lethal phenotype (Lezhneva et al., 2004; Stöckel and Oelmuller, 2004). In the $h c f 101$ mutants, the levels of $4 \mathrm{Fe}-4 \mathrm{~S}$ proteins PsaA, PsaB, and $\mathrm{PsaC}$ were $<4 \%$ of those in the wild type (Lezhneva et al., 2004). In addition, the content of Fd-thioredoxin reductase (FTR, EC 1.8.7.2), another plastidial $4 \mathrm{Fe}-4 \mathrm{~S}$ protein, was significantly reduced in the hcf101 mutants. On the contrary, the amount of Rieske-type $2 \mathrm{Fe}-2 \mathrm{~S}$ protein $\mathrm{PetC}$ was not reduced in the $h c f 101$ mutants and the amount of classic $2 \mathrm{Fe}-2 \mathrm{~S}$ protein $\mathrm{Fd}$ was actually increased. Taken together, these data show that HCF101 is required in the assembly and transfer of $4 \mathrm{Fe}-4 \mathrm{~S}$ (Figure 4A), but not classic or Rieske-type 2Fe-2S (Lezhneva et al., 2004) (Figure 4B). Because the amounts of representative $3 \mathrm{Fe}-4 \mathrm{~S}$ proteins were not determined in the $h c f 101$ mutants, it is not clear whether HCF101 can serve as a carrier protein for $3 \mathrm{Fe}-4 \mathrm{~S}$.

\section{COG0354p}

COG0354 is a family of proteins classified in the Clusters of Orthologous Groups database (Waller et al., 2010). COG0354 proteins are found in all domains of life. IBA57 in yeast and YgfZ in E. coli are two well-studied COG0354 proteins. Mitochondrion-targeted IBA57 is required for the assembly of Fe-S clusters on mitochondrial aconitase (ACO, EC 4.2.1.3), as well as the activation of radical S-adenosylmethionine enzymes, such as isopentenyl-adenosine A37 tRNA methylthiolase (MiaB, EC 2.8.4.3), biotin synthase (BIO, EC 2.8.1.6), and lipoic acid synthase (LIP, EC 2.8.1.8) (Gelling et al., 2008). These four enzymes contain one $4 \mathrm{Fe}-4 \mathrm{~S}$, two $4 \mathrm{Fe}-4 \mathrm{~S}$, one classic $2 \mathrm{Fe}-2 \mathrm{~S}$ plus one $4 \mathrm{Fe}-4 \mathrm{~S}$, and two $4 \mathrm{Fe}-4 \mathrm{~S}$ clusters, respectively (Balk and Pilon, 2011). IBA57 physically interacted with mitochondrial FeS assembly protein 1 and 2 (ISA1 and ISA2) (Gelling et al., 2008), further demonstrating that COG0354 family proteins are part of the Fe-S assembly machinery. YgfZ, which is targeted to the mitochondria as well, is required for the activities of MiaB and some other Fe-S enzymes (Waller et al., 2010).

Plants have two COG0354 proteins: one is plastid-targeted (COG0354p; Figure 3; Table 1) and the other is mitochondriontargeted (COG0354m) (Waller et al., 2010, 2012). Their subcellular localization was confirmed with import assays and confocal microscopic analysis of GFP fusion proteins (Waller et al., 2012). Both COG0354p and COG0354m were capable of complementing the growth defects of $E$. coli $\triangle \mathrm{YgfZ}$ and $\triangle \mathrm{MiaB}$ mutants (Waller et al., 2010, 2012). Similar to IBA57 and YgfZ, the activity of COG0354p and COG0354m is folatedependent (Waller et al., 2012). Inactivation of COG0354m resulted in embryo lethality, indicating that COG0354m is an essential protein (Waller et al., 2012). The phenotype of lossof-function mutants of COG0354p has not yet been described (Table 1). Therefore, it is not clear which Fe-S clusters are carried by COG0354p and COG0354m. However, because inactivation of IBA57 affects $4 \mathrm{Fe}-4 \mathrm{~S}$ and classic $2 \mathrm{Fe}-2 \mathrm{~S}$ enzymes (Gelling et al., 2008), it is possible that COG0354p and COG0354m are involved in the assembly and transfer of $4 \mathrm{Fe}-4 \mathrm{~S}$ and/or classic $2 \mathrm{Fe}-2 \mathrm{~S}$ clusters (Figure 4A).

\section{GRXS14 and GRXS16}

Higher plants have two plastidial GRXs: GRXS14 and GRXS16 (Figure 3; Table 1) (Bandyopadhyay et al., 2008). They were previously thought as activators of vacuolar $\mathrm{H}^{+} / \mathrm{Ca}^{2+}$ antiporters (Cheng and Hirschi, 2003). However, in silico analysis showed that full-length GRXS14 and GRXS16 contain a plastid transit peptide and a GRX domain with a CGFS active site motif (Cheng et al., 2006; Bandyopadhyay et al., 2008). The plastidial localization of GRXS14 and GRXS16 was confirmed by confocal microscopic analysis of GFP fusion proteins. After careful examination of the subcellular localization and physiological function, Cheng et al. (2006) reported that GRXS14 and GRXS16 do not act as activators of vacuolar $\mathrm{H}^{+} / \mathrm{Ca}^{2+}$ antiporters.

Both GRXS14 and GRXS16 were able to complement the growth defects of yeast grx 5 mutant, suggesting that these two proteins are functional GRXs (Bandyopadhyay et al., 2008). Analytical, absorption, and circular dichroism spectral data showed that GRXS14 and GRXS16 bind to one 2Fe-2S cluster per homodimer via the CGFS active site (Table 2) and the binding is assisted by the Cys residues of two glutathione molecules (Bandyopadhyay et al., 2008). In addition, apo GRXS14 served as the scaffold protein during Cys desulfurase-mediated in vitro $2 \mathrm{Fe}-2 \mathrm{~S}$ cluster assembly and the $2 \mathrm{Fe}-2 \mathrm{~S}$ cluster in GRXS14 could be readily transferred to apo Fd (Figure 4A) (Bandyopadhyay et al., 2008). Furthermore, a unidirectional and intact transfer of 2Fe-2S clusters from GRXS14 to SufA1 was observed, suggesting that GRXS14 may work upstream of SufA1 during in vivo delivery of $2 \mathrm{Fe}-2 \mathrm{~S}$ to recipient proteins (Figure 4C) (Mapolelo et al., 2013). It should be noticed that introducing GRXS14 and GRXS16 into yeast grx5 mutant also restores the activity of mitochondrial ACO (Bandyopadhyay et al., 2008). Because mitochondrial ACO requires a $4 \mathrm{Fe}-4 \mathrm{~S}$ cluster to function, it is possible that GRXS14 and GRXS16 act as $4 \mathrm{Fe}-4 \mathrm{~S}$ carriers as well (Figure 4A) (Rouhier et al., 2010). 
In Arabidopsis, GRXS14 and GRXS16 have a similar expression profile: both are expressed in green photosynthetic tissues and flowers (Rey et al., 2017). Consistent with the expression profile, GRXS14 knockout lines and GRXS16 knockdown lines did not exhibit any phenotypic defects under standard growth conditions (Rey et al., 2017). However, the combination of GRXS14 knockout and GRXS16 knockdown resulted in retarded growth, suggesting that GRXS14 and GRXS16 have an overlapping role in the plastid.

Environmental constraints, such as oxidative stress, prolonged darkness, high irradiance, and high salt, change cellular redox status (Rey et al., 2017). Under oxidative stress, GRXS14 knockout lines displayed growth defects in early seedlings (Cheng et al., 2006). Prolonged darkness causes GRXS14 oxidation and reduced contents of proteins involved in maturation of $\mathrm{Fe}-\mathrm{S}$ proteins (Rey et al., 2017). Under prolonged darkness, GRXS14 knockout lines exhibited accelerated chlorophyll loss. These data suggest that GRXS14 is important in protecting proteins again oxidative stress during early seedling growth and maintaining the chlorophyll level under prolonged darkness. Interestingly, GRXS14 overexpression lines demonstrated a reduce chlorophyll content under standard, high irradiance, and high salt conditions (Rey et al., 2017). Therefore, Rey et al. (2017) proposed that GRXS14 may regulate the redox status of chlorophyll biosynthetic enzymes and/or participate in the assembly and transfer of $2 \mathrm{Fe}-2 \mathrm{~S}$ in the plastid, especially under stress conditions. A number of enzymes involved in chlorophyll metabolism contain Fe-S clusters as cofactors: chlorophyllide $a$ oxygenase (CAO, EC 1.14.13.122), 7-hydroxymethyl chlorophyll $a$ reductase (HCAR, EC 1.17.7.2), and pheophorbide $a$ oxygenase (PAO, EC 1.14.15.17) (Oster et al., 2000; Pruzinská et al., 2003; Meguro et al., 2011). Plastidial GRXs may control the redox status of chlorophyll biosynthetic enzymes by regulating the assembly and transfer pathway of Fe-S clusters in the plastids. Consistent with this hypothesis, GRXs, including GRXS14 and GRXS16, could interact with the BolA domain of SufE1 (Table 2) and thereby deglutathionate SufE1, which facilities the activation of plastidial Cys desulfurase (Couturier et al., 2014).

GRXS16 has an N-terminal GlyIleTyr-TyrIleGly (GIY-YIG) endonuclease domain (Figure 3), which confers GRXS16 unique functions, such as coordinating redox regulation and DNA cleavage (Liu et al., 2013). The GIY-YIG domain of GRXS16 alone has endonuclease activity and the activity is decreased substantially in GRXS16 (Liu et al., 2013). On the other hand, the presence of the GIY-YIG domain reduces the ability of GRXS16 to suppress the susceptibility of yeast grx5 mutant to oxidative stress. These data suggest that the two functional domains in GRXS16 negatively regulate each other. Furthermore, Liu et al. (2013) found that Cys ${ }^{123}$ in the GIY-YIG domain may form an intramolecular disulfide bond with Cys $^{219}$ in the CGFS motif of the GRX domain, which explains the negative regulation between the two functional domains.

\section{At-NEET}

As mentioned previously, At-NEET has a CDGSH motif (Figure 3; Table 1), which contains the three Cys and one
His residues necessary for NEET-type $2 \mathrm{Fe}-2 \mathrm{~S}$ coordination (Nechushtai et al., 2012; Su et al., 2013). The first two Cys residues $\left(\mathrm{C}^{74}\right.$ and $\mathrm{C}^{76}$ ) have been shown to be essential for cluster coordination ( $\mathrm{Su}$ et al., 2013), while the His residue $\left(\mathrm{H}^{89}\right)$ has been shown to be essential for the transferability of the 2Fe-2S cluster (Nechushtai et al., 2012). At-NEET forms homodimers (Table 2) and each homodimer coordinates two labile $2 \mathrm{Fe}-2 \mathrm{~S}$ clusters, which are readily transferred to apo Fd in in vivo assays (Nechushtai et al., 2012). Knockdown and RNAi lines of At-NEET showed delayed growth and development, accelerated senescence, and elevated ROS accumulation under standard conditions, increased sensitivity to low $\mathrm{Fe}$ conditions, and reduced sensitivity to high $\mathrm{Fe}$ conditions (Nechushtai et al., 2012). These data suggest that At-NEET is important in Fe metabolism, ROS homeostasis, as well as plant growth, development, and senescence. Therefore, At-NEET might be a carrier protein for the assembly and transfer of NEET-type 2Fe-2S clusters (Nechushtai et al., 2012).

\section{REGULATION OF THE PLASTIDIAL Fe-S ASSEMBLY AND TRANSFER PATHWAY}

Due to the toxicity of free $\mathrm{Fe}$, the biosynthesis of $\mathrm{Fe}$ $S$ clusters has to be regulated together with $\mathrm{Fe}$ uptake (Balk and Schaedler, 2014). Hierarchical clustering analysis of Arabidopsis genes involved in Fe-S assembly and transfer pathways with publicly available microarray data showed that genes for the plastidial SUF pathway form one cluster and genes for the mitochondrial ISC pathway and the cytosolic CIA pathway form another (Balk and Schaedler, 2014). For example, the SufA1, SufB, SufC, SufD, and SufE1 genes cluster together, possibly due to their high expression in photosynthetic tissues (Balk and Schaedler, 2014). These observations suggest that the expression of SUF genes is coordinated and that the plastidial SUF pathway and the ISC/CIA pathways might be differentially regulated. The latter is consistent with the relative independency of the SUF pathway from the ISC/CIA pathways (Van Hoewyk et al., 2007; Bernard et al., 2013).

Balk and Schaedler (2014) also found that under pathogen infection (Pseudomonas syringae) or oxygen deficiency, the expression of ISC and SUF genes is down-regulated (Balk and Schaedler, 2014). Following recognition of the pathogen, the host plant immediately up-regulates ROS production, as part of the defense responses (Torres et al., 2006; Torres, 2010). The rapid rise in ROS tends to destroy Fe-S clusters (Balk and Schaedler, 2014). Therefore, down-regulation of Fe-S cluster assembly at the transcriptional level could be viewed as a preventive method to minimize the content of toxic free Fe released from Fe-S clusters (Balk and Schaedler, 2014).

Several studies showed that $S u f B$ gene expression in Arabidopsis is down-regulated under Fe-deficient conditions (Xu et al., 2005; Balk and Schaedler, 2014). This trend was also observed in rice (Liang et al., 2014). Because SufB is an essential Fe-S scaffold protein (Nagane et al., 2010), a decrease in SufB 
gene expression may quickly decrease the overall rate of $\mathrm{Fe}$ S synthesis in the plastid (Balk and Schaedler, 2014). Further studies are needed to understand the molecular mechanism for Fe deficiency-induced down-regulation of SufB gene expression.

Among Fe-S assembly-related genes, SufE2 was very responsive to a wide range of environmental stimuli (Balk and Schaedler, 2014). As mentioned above, SufE2 only has a single SufE domain, unlike SufE1 and SufE3, which have an additional BolA and NadA domain, respectively (Murthy et al., 2007). Therefore, Balk and Schaedler (2014) proposed that SufE2 may regulate the flux of persulfide $\left(\mathrm{R}-\mathrm{S}-\mathrm{S}^{0} \mathrm{H}\right)$ groups between BolA, the C-terminal domain of SufE1, and NadA, the C-terminal domain of SufE3.

As mentioned above, the two plastid-targeted GRX proteins, GRXS14 and GRXS16, have two Fe-S assembly-related roles. On one hand, GRXS14 and GRXS16 serve as Fe-S carriers in the plastidial Fe-S assembly and transfer pathway (Bandyopadhyay et al., 2008). On the other hand, GRXS14 and GRXS16 are important regulators of redox homeostasis inside the plastid (Rey et al., 2017). GRXS14 and GRXS16 were found to interact with the BolA domain of SufE1 (Couturier et al., 2014). These observations indicate that SufE1 activity and overall Fe-S cluster assembly in the plastid may be subject to redox regulation by GRXS14 and GRXS16 (Couturier et al., 2014). In addition, the interactions between the SufE1 BolA domain and the two plastidial GRX proteins suggest potential link or regulation between the upstream persulfide (R-S- $S^{0} \mathrm{H}$ ) flux at the SufSE complex and the downstream Fe-S transfer steps via Fe-S carrier proteins.

Another protein with a potential regulatory role is At-NEET, a NEET-type 2Fe-2S-containing protein dually targeted to plastids and mitochondria (Nechushtai et al., 2012; Su et al., 2013). As mentioned in a previous paragraph, knockdown and RNAi of AtNEET expression resulted in delayed growth and developmental retardation, enhanced senescence, and increased ROS under standard conditions (Nechushtai et al., 2012). The same mutants also displayed elevated sensitivity to low Fe and decreased sensitivity to high Fe (Nechushtai et al., 2012). Therefore, Nechushtai et al. (2012) concluded that At-NEET is important in Fe metabolism and ROS homeostasis. Additional studies are needed to investigate whether and how At-NEET plays a role in regulating $\mathrm{Fe}-\mathrm{S}$ assembly.

\section{CONCLUDING REMARKS AND OUTSTANDING QUESTIONS}

$\mathrm{Fe}-\mathrm{S}$ clusters and proteins are essential to many biological processes. They are found in the plastids, mitochondria, cytosol, and nucleus of plant cells. Plastidial Fe-S clusters are assembled by the SUF pathway, which consists of Cys desulfurase NFS2, sulfur transferases SufE1, SufE2, and SufE3, SufBC 2 D scaffold complex, and carrier proteins. Cys is the $\mathrm{S}$ source for in vivo assembly of Fe-S clusters. However, it is not yet known what the in vivo $\mathrm{Fe}$ source is and how $\mathrm{Fe}$ is delivered to the scaffold complex. The NFS2-SufE1/2/3-SufBC 2 D system is responsible for de novo assembly of all plastidial Fe-S clusters, because no other Fe-S cluster assembly pathway has been found in the plastid. Single loss-of-function mutations of the NFS2-SufE1/2/3$\mathrm{SufBC}_{2} \mathrm{D}$ system often result in an embryo- or seedling-lethal phenotype, suggesting that the NFS2-SufE1/2/3-SufBC 2 D system is essential. After assembly, Fe-S clusters need to be delivered to appropriate recipient proteins via carrier proteins. A total of nine potential plastidial Fe-S carriers have been identified thus far: SufA1, NFU1, NFU2, NFU3, HCF101, COG0354p, GRXS14, GRXS16, and At-NEET. SufA1, NFU2, GRXS14, and GRXS16 have been shown to act as carrier proteins transferring classic $2 \mathrm{Fe}-2 \mathrm{~S}$ from the $\mathrm{SufBC}_{2} \mathrm{D}$ scaffold complex to recipient proteins. NFU3 has been shown to act as the carrier protein for $3 \mathrm{Fe}-4 \mathrm{~S}$. NFU2, NFU3, and HCF101 have been shown to act as 4Fe-4S carriers. At-NEET is a possible carrier protein for NEET-type $2 \mathrm{Fe}-2 \mathrm{~S}$.

Although NFU1 is a functional NFU protein, it is not clear which $\mathrm{Fe}-\mathrm{S}$ clusters are transferred by this protein. It is possible that NFU1 plays a role in transferring $2 \mathrm{Fe}-2 \mathrm{~S}, 3 \mathrm{Fe}-4 \mathrm{~S}$, and/or $4 \mathrm{Fe}-4 \mathrm{~S}$ clusters because this protein contains a redox-active NFU domain with a conserved CXXC motif capable of coordinating $2 \mathrm{Fe}-2 \mathrm{~S}, 3 \mathrm{Fe}-4 \mathrm{~S}$, and $4 \mathrm{Fe}-4 \mathrm{~S}$ clusters. Functional analysis of recombinant NFU1 protein as well as immunodetection and activity assays of representative plastidial $\mathrm{Fe}-\mathrm{S}$ proteins in lossof-function $n f u 1$ mutants will help address these questions. The types of $\mathrm{Fe}-\mathrm{S}$ clusters COG0354p transfers are also elusive. It is possible that COG0354p plays a role in transferring $4 \mathrm{Fe}-4 \mathrm{~S}$ and classic $2 \mathrm{Fe}-2 \mathrm{~S}$ clusters, because inactivation of COG0354 family protein IBA57 reduces the activities of $4 \mathrm{Fe}-4 \mathrm{~S}$ and classic 2Fe-2S-containing enzymes.

AT-NEET is listed as a carrier protein for NEET-type $2 \mathrm{Fe}$ $2 \mathrm{~S}$. The recombinant AT-NEET homodimer contains two labile $2 \mathrm{Fe}-2 \mathrm{~S}$ clusters, which can be transferred to apo $\mathrm{Fd}$ in vitro (Nechushtai et al., 2012). However, whether plant-type Fds act as an in vivo recipient protein for NEET-type $2 \mathrm{Fe}-2 \mathrm{~S}$ from ATNEET is still questionable, because plant-type Fds are known as classic $2 \mathrm{Fe}-2 \mathrm{~S}$ proteins. Additional experiments are necessary to identify the in vivo recipient proteins for NEET-type $2 \mathrm{~F}-2 \mathrm{~S}$ and to test whether AT-NEET is capable of transferring NEET-type $2 \mathrm{Fe}-2 \mathrm{~S}$ to recipient proteins under in vivo conditions.

It is not yet known which plastidial carriers are responsible for transferring Rieske-type $2 \mathrm{Fe}-2 \mathrm{~S}$ to recipient proteins. NFU1 does not seem to be a good candidate because it does not contain the appropriate binding domain ( $\underline{\mathrm{CX}} \underline{\mathrm{HXGCX}} 12-44 \underline{\mathrm{CXCH}})$ for Rieske-type 2Fe-2S. Although it is well-established that GRXS14 and GRXS16 transfer classic 2Fe-2S, it is possible that these two GRXs may transfer other Fe-S clusters as well. The expression of plastidial GRXS14 and GRXS16 in yeast grx 5 mutant restores the activity of mitochondrial ACO, whose function requires $4 \mathrm{Fe}$ 4S. These results suggest that GRXS14 and GRXS16 may act as carrier proteins for $4 \mathrm{Fe}-4 \mathrm{~S}$ as well. Functional analysis of recombinant GRXS14 and GRXS16 as well as immunodetection and activity assays of representative plastidial $4 \mathrm{Fe}-4 \mathrm{~S}$ proteins in loss-of-function grxs14 and grxs16 mutants will help test this hypothesis.

$3 \mathrm{Fe}-4 \mathrm{~S}$ and $4 \mathrm{Fe}-4 \mathrm{~S}$ have similar absorption spectra, with a broad peak around $410 \mathrm{~nm}$ (Kennedy et al., 1984; Nakamaru-Ogiso et al., 2002). Therefore, it is challenging 
to distinguish the specific types of Fe-S clusters transferred by a Fe-S carrier protein simply via absorption spectral characterization of as-purified and reconstituted $\mathrm{Fe}-\mathrm{S}$ carrier proteins. Immunodetection and activity assays of representative plastidial proteins for each type of Fe-S clusters in loss-of-function mutants will provide complementary information.

Furthermore, the unidirectional and intact transfer of Fe-S clusters from one carrier to another raises the possibly that some $\mathrm{Fe}-\mathrm{S}$ carriers may work together in a sequence when delivering $\mathrm{Fe}-\mathrm{S}$ clusters to the appropriate recipient proteins.

Lastly, although several possible regulation routes have been proposed for the SUF pathway, it is a relatively understudied

\section{REFERENCES}

Abdel-Ghany, S. E., Ye, H., Garifullina, G. F., Zhang, L., Pilon-Smits, E. A., and Pilon, M. (2005). Iron-sulfur cluster biogenesis in chloroplasts. Involvement of the scaffold protein CpIscA. Plant Physiol. 138, 161-172. doi: $10.1104 / \mathrm{pp} .104 .058602$

Ahn, C. S., Lee, J. H., and Pai, H. S. (2005). Silencing of NbNAP1 encoding a plastidic SufB-like protein affects chloroplast development in Nicotiana benthamiana. Mol. Cells 20, 112-118.

Aldea, M., Hernandez-Chico, C., de la Campa, A. G., Kushner, S. R., and Vicente, M. (1988). Identification, cloning, and expression of bolA, an ftsZdependent morphogene of Escherichia coli. J. Bacteriol. 170, 5169-5176. doi: 10.1128/jb.170.11.5169-5176.1988

Ayala-Castro, C., Saini, A., and Outten, F. W. (2008). Fe-S cluster assembly pathways in bacteria. Microbiol. Mol. Biol. Rev. 72, 110-125. doi: 10.1128/MMBR.00034-07

Balk, J., and Pilon, M. (2011). Ancient and essential: the assembly of iron-sulfur clusters in plants. Trends Plant Sci. 16, 218-226. doi: 10.1016/j.tplants.2010.12.006

Balk, J., and Schaedler, T. A. (2014). Iron cofactor assembly in plants. Annu. Rev. Plant Biol. 65, 125-153. doi: 10.1146/annurev-arplant-050213-035759

Bandyopadhyay, S., Gama, F., Molina-Navarro, M. M., Gualberto, J. M., Claxton, R., Naik, S. G., et al. (2008). Chloroplast monothiol glutaredoxins as scaffold proteins for the assembly and delivery of $[2 \mathrm{Fe}-2 \mathrm{~S}]$ clusters. EMBO J. 27, 1122-1133. doi: 10.1038/emboj.2008.50

Beinert, H. (2000). Iron-sulfur proteins: ancient structures, still full of surprises. J. Biol. Inorg. Chem. 5, 2-15. doi: 10.1007/s007750050002

Bernard, D. G., Cheng, Y., Zhao, Y., and Balk, J. (2009). An allelic mutant series of ATM3 reveals its key role in the biogenesis of cytosolic iron-sulfur proteins in Arabidopsis. Plant Physiol. 151, 590-602. doi: 10.1104/pp.109.143651

Bernard, D. G., Netz, D. J., Lagny, T. J., Pierik, A. J., and Balk, J. (2013). Requirements of the cytosolic iron-sulfur cluster assembly pathway in Arabidopsis. Phil. Trans. R. Soc. B 368:20120259. doi: 10.1098/rstb.2012.0259

Briat, J.-F., and Lobréaux, S. (1997). Iron transport and storage in plants. Trends Plant Sci. 2, 187-193. doi: 10.1016/S1360-1385(97)85225-9

Cheng, N. H., and Hirschi, K. D. (2003). Cloning and characterization of CXIP1, a novel PICOT domain-containing Arabidopsis protein that associates with CAX1. J. Biol. Chem. 278, 6503-6509. doi: 10.1074/jbc.M210883200

Cheng, N.-H., Liu, J.-Z., Brock, A., Nelson, R. S., and Hirschi, K. D. (2006). AtGRXcp, an Arabidopsis chloroplastic glutaredoxin, is critical for protection against protein oxidative damage. J. Biol. Chem. 281, 26280-26288. doi: 10.1074/jbc.M601354200

Coschigano, K. T., Melo-Oliveira, R., Lim, J., and Coruzzi, G. M. (1998). Arabidopsis gls mutants and distinct Fd-GOGAT genes. Implications for photorespiration and primary nitrogen assimilation. Plant Cell 10, 741-752. doi: $10.2307 / 3870661$

Couturier, J., Touraine, B., Briat, J. F., Gaymard, F., and Rouhier, N. (2013). The iron-sulfur cluster assembly machineries in plants: current knowledge and open questions. Front. Plant Sci. 4:259. doi: 10.3389/fpls.2013.00259 area. Further investigations are needed to gain a more holistic understanding of the regulatory network of Fe-S assembly and transfer pathways in plants.

\section{AUTHOR CONTRIBUTIONS}

The author confirms being the sole contributor of this work and approved it for publication.

\section{ACKNOWLEDGMENTS}

This work was supported by US National Science Foundation Grant MCB-1244008.

Couturier, J., Wu, H. C., Dhalleine, T., Pegeot, H., Sudre, D., Gualberto, J. M., et al. (2014). Monothiol glutaredoxin-BolA interactions: redox control of Arabidopsis thaliana BolA2 and SufE1. Mol. Plant 7, 187-205. doi: $10.1093 / \mathrm{mp} / \mathrm{sst} 156$

Crack, J. C., Jervis, A. J., Gaskell, A. A., White, G. F., Green, J., Thomson, A. J., et al. (2008). Signal perception by FNR: the role of the iron-sulfur cluster. Biochem. Soc. Trans. 36, 1144-1148. doi: 10.1042/BST0361144

Crane, B. R., and Getzoff, E. D. (1996). The relationship between structure and function for the sulfite reductases. Curr. Opin. Struc. Biol. 6, 744-756 doi: 10.1016/S0959-440X(96)80003-0

Crane, B. R., Siegel, L. M., and Getzoff, E. D. (1995). Sulfite reductase structure at 1.6 Å: evolution and catalysis for reduction of inorganic anions. Science 270, 59-67. doi: 10.1126/science.270.5233.59

Cupp-Vickery, J. R., Urbina, H., and Vickery, L. E. (2003). Crystal structure of IscS, a cysteine desulfurase from Escherichia coli. J. Mol. Biol. 330, 1049-1059. doi: 10.1016/S0022-2836(03)00690-9

Dai, Y., and Outten, F. W. (2012). The E. coli SufS-SufE sulfur transfer system is more resistant to oxidative stress than IscS-IscU. FEBS Lett. 586, 4016-4022. doi: 10.1016/j.febslet.2012.10.001

Dos Santos, P. C., Smith, A. D., Frazzon, J., Cash, V. L., Johnson, M. K., and Dean, D. R. (2004). Iron-sulfur cluster assembly: NifU-directed activation of the nitrogenase Fe protein. J. Biol. Chem. 279, 19705-19711. doi: $10.1074 /$ jbc.M400278200

Foster, J. W., and Moat, A. G. (1980). Nicotinamide adenine dinucleotide biosynthesis and pyridine nucleotide cycle metabolism in microbial systems. Microbiol. Rev. 44, 83-105.

Frazzon, A. P., Ramirez, M. V., Warek, U., Balk, J., Frazzon, J., Dean, D. R., et al. (2007). Functional analysis of Arabidopsis genes involved in mitochondrial iron-sulfur cluster assembly. Plant Mol. Biol. 64, 225-240. doi: $10.1007 /$ s11103-007-9147-x

Frazzon, J., and Dean, D. R. (2003). Formation of iron-sulfur clusters in bacteria: an emerging field in bioinorganic chemistry. Curr. Opin. Chem. Biol. 7, 166-173. doi: 10.1016/S1367-5931(03)00021-8

Gao, H., Subramanian, S., Couturier, J., Naik, S. G., Kim, S. K., Leustek, T., et al. (2013). Arabidopsis thaliana Nfu2 accommodates [2Fe-2S] or [4Fe$4 \mathrm{~S}$ ] clusters and is competent for in vitro maturation of chloroplast [2Fe$2 \mathrm{~S}]$ and [4Fe-4S] cluster-containing proteins. Biochemistry 52, 6633-6645. doi: $10.1021 /$ bi4007622

Gelling, C., Dawes, I. W., Richhardt, N., Lill, R., and Muhlenhoff, U. (2008). Mitochondrial Iba57p is required for $\mathrm{Fe} / \mathrm{S}$ cluster formation on aconitase and activation of radical SAM enzymes. Mol. Cell. Biol. 28, 1851-1861. doi: 10.1128/MCB.01963-07

Hanke, G., and Mulo, P. (2013). Plant type ferredoxins and ferredoxindependent metabolism. Plant Cell Environ. 36, 1071-1084. doi: 10.1111/pce. 12046

Hase, T., Schürmann, P., and Knaff, D. B. (2006). "The interaction of ferredoxin with ferredoxin-dependent enzymes," in Photosystem I: The Light-Driven Plastocyanin:Ferredoxin Oxidoreductase, ed J. H. Golbeck (Dordrecht: Springer Netherlands), 477-498. 
Heidenreich, T., Wollers, S., Mendel, R. R., and Bittner, F. (2005). Characterization of the NifS-like domain of ABA3 from Arabidopsis thaliana provides Insight into the mechanism of molybdenum cofactor sulfuration. J. Biol. Chem. 280, 4213-4218. doi: 10.1074/jbc.M411195200

Hjorth, E., Hadfi, K., Zauner, S., and Maier, U. G. (2005). Unique genetic compartmentalization of the SUF system in cryptophytes and characterization of a SufD mutant in Arabidopsis thaliana. FEBS Lett. 579, 1129-1135. doi: 10.1016/j.febslet.2004.12.084

Holm, R. H., and Lo, W. (2016). Structural conversions of synthetic and protein-bound iron-sulfur clusters. Chem. Rev. 116, 13685-13713. doi: 10.1021/acs.chemrev.6b00276

Hu, X., Kato, Y., Sumida, A., Tanaka, A., and Tanaka, R. (2017a). The SUFBC 2 D complex is required for the biogenesis of all major classes of plastid Fe-S proteins. Plant J. 90, 235-248. doi: 10.1111/tpj.13483

Hu, X., Page, M. T., Sumida, A., Tanaka, A., Terry, M. J., and Tanaka, R. (2017b). The iron-sulfur cluster biosynthesis protein SUFB is required for chlorophyll synthesis, but not phytochrome signaling. Plant J. 89, 1184-1194. doi: $10.1111 /$ tpj. 13455

Jacobson, M. R., Cash, V. L., Weiss, M. C., Laird, N. F., Newton, W. E., and Dean, D. R. (1989). Biochemical and genetic analysis of the nifUSVWZM cluster from Azotobacter vinelandii. Mol. Gen. Genet. 219, 49-57. doi: 10.1007/BF00261156

Johnson, D. C., Dean, D. R., Smith, A. D., and Johnson, M. K. (2005). Structure, function, and formation of biological iron-sulfur clusters. Annu. Rev. Biochem. 74, 247-281. doi: 10.1146/annurev.biochem.74.082803.133518

Kaiser, J. T., Clausen, T., Bourenkow, G. P., Bartunik, H.-D., Steinbacher, S., and Huber, R. (2000). Crystal structure of a NifS-like protein from Thermotoga maritima: implications for iron sulphur cluster assembly. J. Mol. Biol. 297, 451-464. doi: 10.1006/jmbi.2000.3581

Katoh, A., Uenohara, K., Akita, M., and Hashimoto, T. (2006). Early steps in the biosynthesis of NAD in Arabidopsis start with aspartate and occur in the plastid. Plant Physiol. 141, 851-857. doi: 10.1104/pp.106.081091

Kennedy, M. C., Kent, T. A., Emptage, M., Merkle, H., Beinert, H., and Munck, E. (1984). Evidence for the formation of a linear [3Fe-4S] cluster in partially unfolded aconitase. J. Biol. Chem. 259, 14463-14471.

Kim, D.-Y., Bovet, L., Kushnir, S., Noh, E. W., Martinoia, E., and Lee, Y. (2006). AtATM3 is involved in heavy metal resistance in Arabidopsis. Plant Physiol. 140, 922-932. doi: 10.1104/pp.105.074146

Kim, S., and Park, S. (2013). Structural changes during cysteine desulfurase CsdA and sulfur acceptor CsdE interactions provide insight into the transpersulfuration. J. Biol. Chem. 288, 27172-27180. doi: 10.1074/jbc.M113.480277

Kounosu, A., Li, Z., Cosper, N. J., Shokes, J. E., Scott, R. A., Imai, T., et al. (2004). Engineering a three-cysteine, one-histidine ligand environment into a new hyperthermophilic archaeal Rieske-type [2Fe-2S] ferredoxin from Sulfolobus solfataricus. J. Biol. Chem. 279, 12519-12528. doi: 10.1074/jbc.M305923200

Kushnir, S., Babiychuk, E., Storozhenko, S., Davey, M. W., Papenbrock, J., De Rycke, R., et al. (2001). A mutation of the mitochondrial ABC transporter Sta1 leads to dwarfism and chlorosis in the Arabidopsis mutant starik. Plant Cell 13, 89-100. doi: 10.1105/tpc.13.1.89

Léon, S., Touraine, B., Briat, J. F., and Lobreaux, S. (2002). The AtNFS2 gene from Arabidopsis thaliana encodes a NifS-like plastidial cysteine desulphurase. Biochem. J. 366, 557-564. doi: 10.1042/bj20020322

Léon, S., Touraine, B., Ribot, C., Briat, J. F., and Lobréaux, S. (2003). Iron-sulphur cluster assembly in plants: distinct NFU proteins in mitochondria and plastids from Arabidopsis thaliana. Biochem. J. 371, 823-830. doi: 10.1042/bj20021946

Lezhneva, L., Amann, K., and Meurer, J. (2004). The universally conserved HCF101 protein is involved in assembly of [4Fe-4S]-cluster-containing complexes in Arabidopsis thaliana chloroplasts. Plant J. 37, 174-185. doi: 10.1046/j.1365-313X.2003.01952.x

Liang, X., Qin, L., Liu, P., Wang, M., and Ye, H. (2014). Genes for iron-sulphur cluster assembly are targets of abiotic stress in rice, Oryza sativa. Plant Cell Environ. 37, 780-794. doi: 10.1111/pce.12198

Lima, C. D. (2002). Analysis of the E. coli NifS CsdB protein at 2.0 A reveals the structural basis for perselenide and persulfide intermediate formation. J. Mol. Biol. 315, 1199-1208. doi: 10.1006/jmbi.2001.5308

Link, T. A. (1999). The structures of Rieske and Rieske-type proteins. Adv. Inorg. Chem. 47, 83-157. doi: 10.1016/S0898-8838(08)60077-X

Liu, X., Liu, S., Feng, Y., Liu, J. Z., Chen, Y., Pham, K., et al. (2013). Structural insights into the N-terminal GIY-YIG endonuclease activity of Arabidopsis glutaredoxin AtGRXS16 in chloroplasts. Proc. Natl. Acad. Sci. U.S.A. 110, 9565-9570. doi: 10.1073/pnas.1306899110

Lobreaux, S., and Briat, J. F. (1991). Ferritin accumulation and degradation in different organs of pea (Pisum sativum) during development. Biochem. J. 274, 601-606. doi: 10.1042/bj2740601

Madueño, F., Napier, J. A., Cejudo, F. J., and Gray, J. C. (1992). Import and processing of the precursor of the Rieske FeS protein of tobacco chloroplasts. Plant Mol. Biol. 20, 289-299. doi: 10.1007/BF00014496

Mapolelo, D. T., Zhang, B., Randeniya, S., Albetel, A. N., Li, H., Couturier, J., et al. (2013). Monothiol glutaredoxins and A-type proteins: partners in Fe-S cluster trafficking. Dalton Trans. 42, 3107-3115. doi: 10.1039/c2dt32263c

Markley, J. L., Kim, J. H., Dai, Z., Bothe, J. R., Cai, K., Frederick, R. O., et al. (2013). Metamorphic protein IscU alternates conformations in the course of its role as the scaffold protein for iron-sulfur cluster biosynthesis and delivery. FEBS Lett. 587, 1172-1179. doi: 10.1016/j.febslet.2013.01.003

Mason, J. R., and Cammack, R. (1992). The electron-transport proteins of hydroxylating bacterial dioxygenases. Annu. Rev. Microbiol. 46, 277-305. doi: 10.1146/annurev.mi.46.100192.001425

Meguro, M., Ito, H., Takabayashi, A., Tanaka, R., and Tanaka, A. (2011). Identification of the 7-hydroxymethyl chlorophyll a reductase of the chlorophyll cycle in Arabidopsis. Plant Cell 23, 3442-3453. doi: 10.1105/tpc.111.089714

Mihara, H., and Esaki, N. (2002). Bacterial cysteine desulfurases: their function and mechanisms. Appl. Microbiol. Biotechnol. 60, 12-23. doi: 10.1007/s00253-002-1107-4

Mihara, H., Fujii, T., Kato, S., Kurihara, T., Hata, Y., and Esaki, N. (2002). Structure of external aldimine of Escherichia coli CsdB, an IscS/NifS homolog: implications for its specificity toward selenocysteine. J. Biochem. 131, 679-685. doi: 10.1093/oxfordjournals.jbchem.a003151

Mihara, H., Kurihara, T., Yoshimura, T., Soda, K., and Esaki, N. (1997). Cysteine sulfinate desulfinase, a NIFS-like protein of Escherichia coli with selenocysteine lyase and cysteine desulfurase activities. Gene cloning, purification, and characterization of a novel pyridoxal enzyme. J. Biol. Chem. 272, 22417-22424. doi: $10.1074 /$ jbc.272.36.22417

Møller, S. G., Kunkel, T., and Chua, N. H. (2001). A plastidic ABC protein involved in intercompartmental communication of light signaling. Genes Dev. 15, 90-103. doi: 10.1101/gad.850101

Murthy, N. U. M., Ollagnier-de-Choudens, S., Sanakis, Y., Abdel-Ghany, S. E., Rousset, C., Ye, H., et al. (2007). Characterization of Arabidopsis thaliana SufE2 and SufE3: functions in chloroplast iron-sulfur cluster assembly and NAD synthesis. J. Biol. Chem. 282, 18254-18264. doi: 10.1074/jbc.M701428200

Nagane, T., Tanaka, A., and Tanaka, R. (2010). Involvement of AtNAP1 in the regulation of chlorophyll degradation in Arabidopsis thaliana. Planta 231, 939-949. doi: 10.1007/s00425-010-1099-8

Nakamaru-Ogiso, E., Yano, T., Ohnishi, T., and Yagi, T. (2002). Characterization of the iron-sulfur cluster coordinated by a cysteine cluster motif (CXXCXXXCX27C) in the Nqo3 subunit in the proton-translocating NADH-quinone oxidoreductase (NDH-1) of Thermus thermophilus HB-8. J. Biol. Chem. 277, 1680-1688. doi: 10.1074/jbc.M108796200

Nath, K., O’Donnell, J. P., and Lu, Y. (2017). Chloroplastic iron-sulfur scaffold protein NFU3 is essential to overall plant fitness. Plant Signal. Behav. 12:e1282023. doi: 10.1080/15592324.2017.1282023

Nath, K., Wessendorf, R. L., and Lu, Y. (2016). A nitrogen-fixing subunit essential for accumulating $4 \mathrm{Fe}-4 \mathrm{~S}$-containing Photosystem I core proteins. Plant Physiol. 172, 2459-2470. doi: 10.1104/pp.16.01564

Nechushtai, R., Conlan, A. R., Harir, Y., Song, L., Yogev, O., Eisenberg-Domovich, Y., et al. (2012). Characterization of Arabidopsis NEET reveals an ancient role for NEET proteins in iron metabolism. Plant Cell 24, 2139-2154. doi: $10.1105 /$ tpc. 112.097634

Oster, U., Tanaka, R., Tanaka, A., and Rudiger, W. (2000). Cloning and functional expression of the gene encoding the key enzyme for chlorophyll b biosynthesis (CAO) from Arabidopsis thaliana. Plant J. 21, 305-310. doi: 10.1046/j.1365-313x.2000.00672.x

Outten, F. W., Djaman, O., and Storz, G. (2004). A suf operon requirement for FeS cluster assembly during iron starvation in Escherichia coli. Mol. Microbiol. 52, 861-872. doi: 10.1111/j.1365-2958.2004.04025.x

Outten, F. W., Wood, M. J., Muñoz, F. M., and Storz, G. (2003). The SufE protein and the SufBCD complex enhance SufS cysteine desulfurase activity as part of 
a sulfur transfer pathway for Fe-S cluster assembly in Escherichia coli. J. Biol. Chem. 278, 45713-45719. doi: 10.1074/jbc.M308004200

Pilon-Smits, E. A., Garifullina, G. F., Abdel-Ghany, S., Kato, S., Mihara, H., Hale, K. L., et al. (2002). Characterization of a NifS-like chloroplast protein from Arabidopsis. Implications for its role in sulfur and selenium metabolism. Plant Physiol. 130, 1309-1318. doi: 10.1104/pp.102.010280

Pruzinská, A., Tanner, G., Anders, I., Roca, M., and Hortensteiner, S. (2003). Chlorophyll breakdown: pheophorbide $a$ oxygenase is a Riesketype iron-sulfur protein, encoded by the accelerated cell death 1 gene. Proc. Natl. Acad. Sci. U.S.A. 100, 15259-15264. doi: 10.1073/pnas.20365 71100

Raux-Deery, E., Leech, H. K., Nakrieko, K. A., McLean, K. J., Munro, A. W., Heathcote, P., et al. (2005). Identification and characterization of the terminal enzyme of siroheme biosynthesis from Arabidopsis thaliana: a plastid-located sirohydrochlorin ferrochelatase containing a $2 \mathrm{Fe}-2 \mathrm{~S}$ center. J. Biol. Chem. 280, 4713-4721. doi: 10.1074/jbc.M411360200

Ravet, K., Touraine, B., Boucherez, J., Briat, J. F., Gaymard, F., and Cellier, F. (2009). Ferritins control interaction between iron homeostasis and oxidative stress in Arabidopsis. Plant J. 57, 400-412. doi: 10.1111/j.1365-313X.2008.03698.x

Rey, P., Becuwe, N., Tourrette, S., and Rouhier, N. (2017). Involvement of Arabidopsis glutaredoxin S14 in the maintenance of chlorophyll content. Plant Cell Environ. 40, 2319-2332. doi: 10.1111/pce.13036

Roche, B., Aussel, L., Ezraty, B., Mandin, P., Py, B., and Barras, F. (2013). Iron/sulfur proteins biogenesis in prokaryotes: formation, regulation and diversity. Biochim. Biophys. Acta 1827, 455-469. doi: 10.1016/j.bbabio.2012.12.010

Roret, T., Pegeot, H., Couturier, J., Mulliert, G., Rouhier, N., and Didierjean, C. (2014). X-ray structures of Nfs2, the plastidial cysteine desulfurase from Arabidopsis thaliana. Acta Crystallogr. Sect. F Struct. Biol. Cryst. Commun. 70, 1180-1185. doi: 10.1107/S2053230X14017026

Rouhier, N., Couturier, J., Johnson, M. K., and Jacquot, J.-P. (2010). Glutaredoxins: roles in iron homeostasis. Trends Biochem. Sci. 35:43. doi: 10.1016/j.tibs.2009.08.005

Saenger, W., Jordan, P., and Krauss, N. (2002). The assembly of protein subunits and cofactors in Photosystem, I. Curr. Opin. Struc. Biol. 12, 244-254. doi: 10.1016/S0959-440X(02)00317-2

Saha, K., Webb, M. E., Rigby, S. E., Leech, H. K., Warren, M. J., and Smith, A. G. (2012). Characterization of the evolutionarily conserved iron-sulfur cluster of sirohydrochlorin ferrochelatase from Arabidopsis thaliana. Biochem. J. 444, 227-237. doi: 10.1042/BJ20111993

Saini, A., Mapolelo, D. T., Chahal, H. K., Johnson, M. K., and Outten, F. W. (2010). SufD and SufC ATPase activity are required for iron acquisition during in vivo Fe-S cluster formation on SufB. Biochemistry 49, 9402-9412. doi: $10.1021 /$ bi1011546

Schwenkert, S., Netz, D. J., Frazzon, J., Pierik, A. J., Bill, E., Gross, J., et al. (2010). Chloroplast HCF101 is a scaffold protein for [4Fe-4S] cluster assembly. Biochem. J. 425, 207-214. doi: 10.1042/BJ20091290

Singh, H., Dai, Y., Outten, F. W., and Busenlehner, L. S. (2013). Escherichia coli SufE sulfur transfer protein modulates the SufS cysteine desulfurase through allosteric conformational dynamics. J. Biol. Chem. 288, 36189-36200. doi: $10.1074 /$ jbc.M113.525709

Stöckel, J., and Oelmuller, R. (2004). A novel protein for Photosystem I biogenesis. J. Biol. Chem. 279, 10243-10251. doi: 10.1074/jbc.M3092 46200

Su, L. W., Chang, S. H., Li, M. Y., Huang, H. Y., Jane, W. N., and Yang, J. Y. (2013). Purification and biochemical characterization of Arabidopsis At-NEET, an ancient iron-sulfur protein, reveals a conserved cleavage motif for subcellular localization. Plant Sci. 213, 46-54. doi: 10.1016/j.plantsci.2013.09.001

Takahashi, Y., Mitsui, A., Fujita, Y., and Matsubara, H. (1991a). Roles of ATP and NADPH in formation of the Fe-S cluster of spinach ferredoxin. Plant Physiol. 95, 104-110. doi: 10.1104/pp.95.1.104

Takahashi, Y., Mitsui, A., Hase, T., and Matsubara, H. (1986). Formation of the iron-sulfur cluster of ferredoxin in isolated chloroplasts. Proc. Natl. Acad. Sci. U.S.A. 83, 2434-2437. doi: 10.1073/pnas.83.8.2434

Takahashi, Y., Mitsui, A., and Matsubara, H. (1991b). Formation of the Fe-S cluster of ferredoxin in lysed spinach chloroplasts. Plant Physiol. 95, 97-103. doi: $10.1104 /$ pp. 95.1 .97
Takahashi, Y., and Nakamura, M. (1999). Functional assignment of the ORF2-iscS-iscU-iscA-hscB-hscA-fdx-ORF3 gene cluster involved in the assembly of $\mathrm{Fe}-\mathrm{S}$ clusters in Escherichia coli. J. Biochem. 126, 917-926. doi: 10.1093/oxfordjournals.jbchem.a0 22535

Takahashi, Y., and Tokumoto, U. (2002). A third bacterial system for the assembly of iron-sulfur clusters with homologs in archaea and plastids. J. Biol. Chem. 277, 28380-28383. doi: 10.1074/jbc.C200365200

Torres, M. A. (2010). ROS in biotic interactions. Physiol. Plant. 138, 414-429. doi: 10.1111/j.1399-3054.2009.01326.x

Torres, M. A., Jones, J. D., and Dangl, J. L. (2006). Reactive oxygen species signaling in response to pathogens. Plant Physiol. 141, 373-378. doi: 10.1104/pp.106.079467

Touraine, B., Boutin, J. P., Marion-Poll, A., Briat, J. F., Peltier, G., and Lobreaux, S. (2004). Nfu2: a scaffold protein required for [4Fe-4S] and ferredoxin ironsulphur cluster assembly in Arabidopsis chloroplasts. Plant J. 40, 101-111. doi: 10.1111/j.1365-313X.2004.02189.x

van den Heuvel, R. H., Ferrari, D., Bossi, R. T., Ravasio, S., Curti, B., Vanoni, M. A., et al. (2002). Structural studies on the synchronization of catalytic centers in glutamate synthase. J. Biol. Chem. 277, 24579-24583. doi: 10.1074/jbc.M202541200

Van Hoewyk, D., Abdel-Ghany, S. E., Cohu, C. M., Herbert, S. K., Kugrens, P., Pilon, M., et al. (2007). Chloroplast iron-sulfur cluster protein maturation requires the essential cysteine desulfurase CpNifS. Proc. Natl. Acad. Sci. U.S.A. 104, 5686-5691. doi: 10.1073/pnas.0700774104

Vigani, G., Maffi, D., and Zocchi, G. (2009). Iron availability affects the function of mitochondria in cucumber roots. New Phytol. 182, 127-136. doi: 10.1111/j.1469-8137.2008.02747.x

Waller, J. C., Alvarez, S., Naponelli, V., Lara-Nunez, A., Blaby, I. K., Da Silva, V., et al. (2010). A role for tetrahydrofolates in the metabolism of iron-sulfur clusters in all domains of life. Proc. Natl. Acad. Sci. U.S.A. 107, 10412-10417. doi: 10.1073/pnas.0911586107

Waller, J. C., Ellens, K. W., Alvarez, S., Loizeau, K., Ravanel, S., and Hanson, A. D. (2012). Mitochondrial and plastidial COG0354 proteins have folatedependent functions in iron-sulphur cluster metabolism. J. Exp. Bot. 63, 403-411. doi: 10.1093/jxb/err286

Wiley, S. E., Murphy, A. N., Ross, S. A., van der Geer, P., and Dixon, J. E. (2007). MitoNEET is an iron-containing outer mitochondrial membrane protein that regulates oxidative capacity. Proc. Natl. Acad. Sci. U.S.A. 104, 5318-5323. doi: 10.1073/pnas.0701078104

Wollers, S., Layer, G., Garcia-Serres, R., Signor, L., Clemancey, M., Latour, J. M., et al. (2010). Iron-sulfur (Fe-S) cluster assembly: the SufBCD complex is a new type of Fe-S scaffold with a flavin redox cofactor. J. Biol. Chem. 285, 23331-23341. doi: 10.1074/jbc.M110.127449

Xu, X. M., Adams, S., Chua, N. H., and Møller, S. G. (2005). AtNAP1 represents an atypical SufB protein in Arabidopsis plastids. J. Biol. Chem. 280, 6648-6654. doi: $10.1074 / j b c . M 413082200$

Xu, X. M., and Møller, S. G. (2004). AtNAP7 is a plastidic SufC-like ATP-binding cassette/ATPase essential for Arabidopsis embryogenesis. Proc. Natl. Acad. Sci. U.S.A. 101, 9143-9148. doi: 10.1073/pnas.0400799101

$\mathrm{Xu}, \mathrm{X}$. M., and Møller, S. G. (2006). AtSufE is an essential activator of plastidic and mitochondrial desulfurases in Arabidopsis. EMBO J. 25, 900-909. doi: 10.1038/sj.emboj.7600968

Yabe, T., Morimoto, K., Kikuchi, S., Nishio, K., Terashima, I., and Nakai, M. (2004). The Arabidopsis chloroplastic NifU-like protein CnfU, which can act as an iron-sulfur cluster scaffold protein, is required for biogenesis of ferredoxin and Photosystem, I. Plant Cell 16, 993-1007. doi: 10.1105/tpc.0 20511

Yabe, T., and Nakai, M. (2006). Arabidopsis AtIscA-I is affected by deficiency of Fe-S cluster biosynthetic scaffold AtCnfU-V. Biochem. Biophys. Res. Commun. 340, 1047-1052. doi: 10.1016/j.bbrc.2005.12.104

Yadav, S., Kushwaha, H. R., Kumar, K., and Verma, P. K. (2012). Comparative structural modeling of a monothiol GRX from chickpea: insight in iron-sulfur cluster assembly. Int. J. Biol. Macromol. 51, 266-273. doi: 10.1016/j.ijbiomac.2012.05.014

Ye, H., Abdel-Ghany, S. E., Anderson, T. D., Pilon-Smits, E. A., and Pilon, M. (2006). CpSufE activates the cysteine desulfurase CpNifS for chloroplastic Fe-S cluster formation. J. Biol. Chem. 281, 8958-8969. doi: 10.1074/jbc.M512737200 
Ye, H., Garifullina, G. F., Abdel-Ghany, S. E., Zhang, L., Pilon-Smits, E. A., and Pilon, M. (2005). The chloroplast NifS-like protein of Arabidopsis thaliana is required for iron-sulfur cluster formation in ferredoxin. Planta 220, 602-608. doi: 10.1007/s00425-004-1388-1

Zhang, B., Crack, J. C., Subramanian, S., Green, J., Thomson, A. J., Le Brun, N. E., et al. (2012). Reversible cycling between cysteine persulfide-ligated [2Fe-2S] and cysteine-ligated [4Fe-4S] clusters in the FNR regulatory protein. Proc. Natl. Acad. Sci. U.S.A. 109, 15734-15739. doi: 10.1073/pnas.12087 87109

Zheng, L., and Dean, D. R. (1994). Catalytic formation of a nitrogenase iron-sulfur cluster. J. Biol. Chem. 269, 18723-18726.

Zheng, L., White, R. H., Cash, V. L., and Dean, D. R. (1994). Mechanism for the desulfurization of L-cysteine catalyzed by the nifS gene product. Biochemistry 33, 4714-4720. doi: 10.1021/bi00181a031
Zheng, L., White, R. H., Cash, V. L., Jack, R. F., and Dean, D. R. (1993). Cysteine desulfurase activity indicates a role for NIFS in metallocluster biosynthesis. Proc. Natl. Acad. Sci. U.S.A. 90, 2754-2758. doi: 10.1073/pnas.90.7.2754

Conflict of Interest Statement: The author declares that the research was conducted in the absence of any commercial or financial relationships that could be construed as a potential conflict of interest.

Copyright $\odot 2018 \mathrm{Lu}$. This is an open-access article distributed under the terms of the Creative Commons Attribution License (CC BY). The use, distribution or reproduction in other forums is permitted, provided the original author(s) and the copyright owner are credited and that the original publication in this journal is cited, in accordance with accepted academic practice. No use, distribution or reproduction is permitted which does not comply with these terms. 